\title{
INFINITE TOWERS OF ARTIN-SCHREIER DEFECT EXTENSIONS OF RATIONAL FUNCTION FIELDS
}

\author{
ANNA BLASZCZOK
}

\begin{abstract}
We consider Artin-Scheier defect extensions of rational function fields in two variables over fields of positive characteristic. We study the problem of constructing infinite towers of such extensions. We classify ArtinSchreier defect extensions into "dependent" and "independent" ones, according to whether they are connected with purely inseparable defect extensions, or not. To understand the meaning of the classification for the issue of local uniformization, we consider various valuations of the rational function field and investigate for which it admits an infinite tower of dependent or independent Artin-Schreier defect extensions. We give also a criterion for a valued field of positive characteristic $p$ with $p$-divisible value group and perfect residue field to admit infnitely many parallel dependent Artin-Schreier defect extensions or an infinite tower of such extensions.
\end{abstract}

\section{INTRODUCTION}

We denote by $(K, v)$ a field $K$ equipped with a (Krull) valuation $v$. Its value group will be denoted by $v K$ and its residue field by $K v$. Writing $(L \mid K, v)$ we mean an extension of valued fields, where $v$ is a valuation of $L$ and $K$ is equipped with the restriction of this valuation. If the canonical embedings of $v K$ in $v L$ and of $K v$ in $L v$ are surjective (which will be expressed by writing $v L=v K$ and $L v=K v$ ) then the extension is called immediate.

Take a valued field $(K, v)$. Every finite extension $L$ of $K$ satisfies the fundamental inequality (cf. $[\mathrm{ZS}],[\mathrm{E}])$ :

$$
n \geq \sum_{i=1}^{g} e_{i} f_{i}
$$

where $n=[L: K]$ is the degree of the extension, $v_{1}, \ldots, v_{g}$ are all distinct extensions of the valuation $v$ from $K$ to $L, e_{i}=\left(v_{i} L: v K\right)$ are the respective ramification indices and $f_{i}=\left[L v_{i}: K v\right]$ are the respective inertia degrees. If the valuation $v$ of $K$ admits a unique extension to the algebraic closure $\tilde{K}$ of $K$, which holds if $g=1$ for every finite extension $L \mid K$, then $K$ is called henselian. There is a minimal separable-algebraic extension of $(K, v)$ which is henselian. This extension is unique up to isomorphism over $K$. It is called the henselisation of $(K, v)$ and denoted by $(K, v)^{h}$ or, if $v$ is fixed, by $K^{h}$.

Date: 20. 11. 2012.

2010 Mathematics Subject Classification. Primary 12J10; Secondary 12J20, 16W60.

I wish to thank Professor Franz-Viktor Kuhlmann for his invaluable help, support and suggestions. Part of this paper was prepared while I was a guest of the Department of Mathematics and Statistics of the University of Saskatchewan. I gratefully acknowledge their hospitality and support. During work on this paper, I was supported by the UPGOW Project grant. 
Take a finite extension $L$ of a valued field $(K, v)$. Then the extension of $v$ to $L$ is unique if and only if $L \mid K$ is linearly disjoint from some henselization of $(K, v)$. In this case, by the Lemma of Ostrowski,

$$
[L: K]=(v L: v K)[L v: K v] \cdot p^{\nu},
$$

where $\nu$ is a nonnegative integer and $p$ the characteristic exponent of $K v$, that is, $p=\operatorname{char} K v$ if it is positive and $p=1$ otherwise. The factor $d(L \mid K, v)=p^{\nu}$ is called the defect of the extension $(L \mid K, v)$. If it is nontrivial, that is, if $\nu>0$, then we call $L \mid K$ a defect extension. If $d(L \mid K, v)=1$ we speak of a defectless extension.

The phenomenon of nontrivial defect, appearing only if the residue characteristic of the valued field is positive, plays often an essential role in problems in algebraic geometry or the model theory of valued fields (see, e.g. [Kn-Ku1], [Kn-Ku2] and $[\mathrm{Ku} 5])$. In Section 4.1 we prove the following fact which is a generalization of Theorem 1.2 of [Ku1]. It shows that even if the field seems to be simple it may admit defect extensions.

Theorem 1.1. Take a field $K$ of positive characteristic $p$ and assume that it admits a perfect subfield of cardinality $\kappa$. Then there is a valuation $v$ on the rational function field $K(x, y) \mid K$ whose restriction to $K$ is trivial, such that $(K(x, y), v)$ admits $\kappa$ many pairwise linearly disjoint infinite towers of Galois extensions of degree and defect $p$.

The structure of defect extensions of rational function fields is especially interesting in connection with problems related to resolution of singularities. The nontrivial defect is one of the hurdles for the attempt to prove local uniformization in positive characteristic. In particular, in the case of two dimensional algebraic function fields of positive characteristic, a strong relative form of local uniformization presented in Theorem 7.35 of [CP] may not hold if positive defect appears. This can be shown by an example which consists of a tower of two Artin-Schreier defect extensions of a rational function field in two variables (cf. Theorem 7.38 of $[\mathrm{CP}]$ ). Recall that an Artin-Schreier extension of a field $K$ of positive characteristic $p$ is an extension of degree $p$ generated by a root $\vartheta$ of a polynomial $X^{p}-X-a$ with $a \in K$. In this case, $\vartheta$ is called an Artin-Schreier generator of the extension. Since the other roots of the polynomial $X^{p}-X-a$ are of the form $\vartheta+1, \ldots, \vartheta+p-1$, such an extension is always normal and hence Galois. On the other hand, every Galois extension of $K$ of degree $p$ is an Artin-Schreier extension (see, e.g., [L]). Therefore, Theorem 1.1 states that there is a valuation $v$ on $K(x, y) \mid K$ such that the field $(K(x, y), v)$ admits infinite towers of Artin-Schreier defect extensions.

The importance of studying the structure of such extensions comes from the fact that towers of Artin-Schreier defect extensions play a central role in the issue of defect extensions. Take a valued field $(K, v)$ of positive characteristic $p$. Denote by $K^{r}$ the absolute ramification field of $K$, i.e., the ramification field of the extension $K^{s e p} \mid K$ with respect to a fixed extension of $v$ to the separable-algebraic closure $K^{\text {sep }}$ of $K$. Take any finite extension $(L \mid K, v)$ such that the extension of the valuation $v$ of $K$ to $L$ is unique. If $L . K^{r}$ is the field compositum of $L$ and $K^{r}$, then the extension $L . K^{r} \mid K^{r}$ has the same defect as $L \mid K$ (cf. Proposition 2.8 of [Ku3]). On the other hand, $L \cdot K^{r} \mid K^{r}$ is a tower of normal extensions of degree $p$ (cf., e.g., Lemma 2.9 of [Ku3]). Thus, if $L \mid K$ is separable, $L . K^{r} \mid K^{r}$ is a tower of Artin-Schreier extensions and if $(L \mid K, v)$ is a defect extension, then so are some of 
these Artin-Schreier extensions. Because of this representative role of Artin-Schreier defect extensions, one would like to understand better their structure. In particular, one would like to know if all of the extensions behave in a similar way in relation to a given problem, like local uniformization. A classification of Artin-Schreier defect extensions presented in [Ku3] considers their connection with purely inseparable extensions. More precisely, if for a given Artin-Schreier defect extension $K(\vartheta) \mid K$ with $\vartheta^{p}-\vartheta \in K$ there is an immediate purely inseparable extension $K(\eta) \mid K$, where $\eta \in K^{1 / p} \backslash K$, such that

$$
v(\vartheta-c)=v(\eta-c)
$$

for every $c \in K$ then $K(\vartheta) \mid K$ is called a dependent Artin-Schreier defect extension. Otherwise we speak of an independent Artin-Schreier defect extension.

There are indications that considering this classification in connection with the problem of local uniformization is meaningful. Temkin's work (especially [T]) shows that the dependent Artin-Schreier defect extensions may be more harmful. The conjecture seems to be affirmed by the mentioned example of Cutkosky and Piltant. Work in progress of Ghezzi, ElHitti and Kuhlmann indicates that the tower of two Artin-Schreier defect extensions considered in the example consist of dependent extensions.

To better understand the meaning of the classification for the problem of local uniformization, it can therefore be helpful to study possible constructions and the structure of towers of dependent and independent Artin-Schreier defect extensions of rational function fields. A hurdle for that is the use of different languages in the constructions and in the description of properties of the extensions. Namely, the language used by algebraic geometers in the constructions of valuations and defect extensions of rational function fields is essentially different from the language used (so far) in the characterizations of dependent and independent Artin-Schreier defect extensions. For instance, the difficulty of proving that the extension in the example of Cutkosky and Piltant consists of two dependent Artin-Schreier defect extensions is connected with the fact that the valuation is given there by means of generating sequences, whereas the criterion for dependence or independence (see Proposition 2.19 ) is closer to Kaplansky's notion of pseudo Cauchy sequences (cf. [Ka], Section 2). The classification of Artin-Schreier defect extensions is also reflected in higher ramification groups of the extensions, which will be worked out in $[\mathrm{Ku}-\mathrm{P}]$. The problem in this case is finding an efficient algorithm that converts between generating sequences, higher ramification groups and pseudo Cauchy sequences.

In this paper we will present examples of towers of both dependent and independent Artin-Schreier defect extensions of rational function fields in two variables using pseudo Cauchy sequences to describe valuations of fields. We show that all infinite towers of Artin-Schreier defect extensions constructed in the proof of Theorem 1.1 consist of independent extensions. Therefore we obtain the following theorem:

Theorem 1.2. Take a field $K$ of positive characteristic and assume that it admits a perfect subfield of cardinality $\kappa$. Then there is a valuation $v$ of the rational function field $K(x, y) \mid K$, trivial on $K$, such that $(K(x, y), v)$ admits $\kappa$ many pairwise linearly disjoint infinite towers of independent Artin-Schreier defect extensions. 
Due to the possible importance of the classification of Artin-Schreier defect extensions for the problems related to local uniformization, an interesting question is whether such constructions are also possible with dependent in the place of independent Artin-Schreier defect extensions. The following theorem gives an answer:

Theorem 1.3. If $K$ is a perfect field of positive characteristic, then there is a valuation $v$ of the rational function field $K(x, y) \mid K$, trivial on $K$, such that $(K(x, y), v)$ admits $\max \left\{|K|, \aleph_{0}\right\}$ many pairwise linearly disjoint infinite towers of dependent Artin-Schreier defect extensions.

On the other hand, in Section 4.2 we give an example of a valuation $v$ on the field $K(x, y)$ satisfying the assumptions of the above theorem, such that $(K(x, y), v)$ admits no dependent, but admits infinite towers of independent Artin-Schreier defect extensions.

In all of these cases we consider valued rational function fields of positive characteristic $p$ with $p$-divisible value groups. We cannot answer yet whether similar constructions are possible in the non- $p$-divisible case. More precisely, we are not yet able to show that the Artin-Schreier extensions we can construct with the use of pseudo Cauchy sequences are linearly disjoint from the henselization.

In Section 3 we give a criterion for a valued field $(K, v)$ of positive characteristic $p$ with $p$-divisible value group and perfect residue field to admit an infinite tower of dependent Artin-Schreier defect extensions.

Theorem 1.4. Take a valued field $(K, v)$ of positive characteristic $p$ with perfect residue field and p-divisible value group. If there is a purely inseparable extension of $K$ which does not lie in the completion of the field, then $K$ admits an infinite tower of dependent Artin-Schreier defect extensions. If every purely inseparable extension of degree $p$ lies in the completion of $K$, then the field admits no dependent Artin-Schreier defect extensions.

We prove that the same condition implies that the field admits infinitely many parallel dependent Artin-Schreier defect extensions, such that the compositum of any $n$ of the extensions is a Galois extension of degree and defect $p^{n}$ (see Proposition 3.2).

In Section 5 we show that a simple generalization of the technique used in the proof of Theorem 1.1 enables us to construct infinite towers of Artin-Schreier defect extensions of rational function fields, using Galois extensions of degree $p^{n}$ generated by roots of polynomials of the form $X^{p^{n}}-X-a$. We give an example that a similar extension of the techniques used for the construction of dependent Artin-Schreier defect extensions may not lead to towers of Artin-Schreier defect extensions. More precisely, Theorem 2.21 states that by a certain deformation of a polynomial inducing an immediate purely inseparable extension of prime degree not contained in the completion of the field we obtain an Artin-Schreier defect extension. Example 5.5 shows that for purely inseparable extensions of higher degrees such deformation may lead to extensions not disjoint from the henselization. The same example shows also that we cannot generalize Theorem 2.14 replacing the condition " $a$ lies in $K^{h}$ " by " $K(a) \mid K$ is not linearly disjoint from $K^{h} \mid K$ ". 


\section{Preliminaries}

For the next results and basic facts of valuation theory we refer the reader to [E], $[R],[W]$ and $[\mathrm{ZS}]$. We assume some familiarity with the theory of pseudo Cauchy sequences as presented in $[\mathrm{Ka}]$, called there "pseudo convergent sets". We will also use the name of "pseudo limit" in place of limit of a pseudo Cauchy sequence.

Take a valued field $(K, v)$. Then any extension of $v$ to the algebraic closure $\tilde{K}$ of $K$ has residue field $\widetilde{K v}$ and value group $\widetilde{v K}$, that is, the divisible hull of $v K$.

\subsection{Linearly disjoint and algebraically disjoint extensions.}

In this section we recall a few properties of linearly and algebraically disjoint extensions. For the proofs and details see for instance $[\mathrm{L}]$ and $[\mathrm{Ku} 6]$.

Lemma 2.1. Take $L \mid K$ and $F \supseteq E \supseteq K$ to be field extensions contained in a common extension field $\Omega$. Then $L \mid K$ is linearly disjoint from $F \mid K$ if and only if $L \mid K$ is linearly disjoint from $E \mid K$ and $L . E \mid E$ is linearly disjoint from $F \mid E$.

The next lemma gives a useful criterion for linear disjointness if at least one of the extensions is Galois.

Lemma 2.2. Suppose $L \mid K$ is a Galois and $F \mid K$ an arbitrary field extension. Then $L$ and $F$ are linearly disjoint over $K$ if and only if $L \cap F=K$.

Using this Lemma and properties of Galois extensions one can show the following fact.

Corollary 2.3. Take an arbitrary field extension $K^{\prime} \mid K$ and a Galois extension $L \mid K$, linearly disjoint from $K^{\prime} \mid K$. Then the extension $L . K^{\prime} \mid K^{\prime}$ is also Galois and the restriction of the automorphisms of $L . K^{\prime} \mid K^{\prime}$ to the field $L$ is a topological isomorphism of $\operatorname{Gal}\left(L . K^{\prime} \mid K^{\prime}\right)$ and $\operatorname{Gal}(L \mid K)$.

A field extension $L \mid K$ will be called separable if it is linearly disjoint from $K^{1 / p^{\infty}} \mid K$, or equivalently, from $K^{1 / p} \mid K$. Note that in the case of algebraic extensions the definition coincides with the standard notion of separable extensions. Such extensions will be called separable-algebraic.

Lemma 2.4. If $F \mid K$ is an arbitrary extension such that $K$ is relatively algebraically closed in $F$, then $F \mid K$ is linearly disjoint form every separable-algebraic extension of $K$.

Using the properties of linearly disjoint extensions we can give an easy proof of the amalgamation property of valued field extensions in the case of simple algebraic and simple transcendental extensions.

Lemma 2.5. Take a valued field $(L, v)$. If $\left(L(b), v_{1}\right)$ is an algebraic extension and $\left(L(x), v_{2}\right)$ a transcendental extension of the field $(L, v)$, then there is an extension $w$ of the valuation $v$ to the field $L(x, b)$ such that the restrictions of $w$ to the fields $L(b)$ and $L(x)$ coincide with $v_{1}$ and $v_{2}$ respectively.

Proof. Let $F$ be an algebraic extension of $L(b)$ such that $F \mid L$ is normal. Take an extension $v_{1}^{\prime}$ of the valuation $v_{1}$ to the field $F$ and an extension $v_{2}^{\prime}$ of $v_{2}$ to the field $L(x) . F=F(x)$. Since $v_{1}^{\prime}$ and $v_{2}^{\prime}$ coincide on $L$, the valuations $v_{1}^{\prime}$ and $\left.v_{2}^{\prime}\right|_{F}$ are conjugate. Take $\sigma \in \operatorname{Gal}(F \mid L)$ such that $v_{1}^{\prime}=\left.v_{2}^{\prime}\right|_{F} \circ \sigma$.

If $b$ is purely inseparable over $L$, one can choose $F=L(b)$ and $F(x) \mid L(x)$ is purely inseparable. Then $\sigma=i d_{L(b)}$. Hence $v_{2}^{\prime}$ is the unique extension of $v_{2}$ to $L(x, b)$ and 
$\left.v_{2}^{\prime}\right|_{L(b)}=v_{1}$. Suppose that the element $b$ is separable over $L$. Then one can take $F$ to be separable extension of $L$ and thus by Lemma 2.4, the extensions $F \mid L$ and $L(x) \mid L$ are linearly disjoint. Since $F \mid L$ is a Galois extension, from Corollary 2.3 it follows that $\sigma$ can be extended to the automorphism $\tilde{\sigma} \in \operatorname{Gal}(F(x) \mid L(x))$. Setting $w:=\left.v_{2}^{\prime} \circ \tilde{\sigma}\right|_{L(x, b)}$ we obtain an extension of the valuations $v_{1}$ and $v_{2}$ to the field $L(x, b)$.

Take a field $K$ and let $L|K, F| K$ be subextensions of some field extension $\Omega$ of the field $K$. The extension $L \mid K$ is called algebraically disjoint from $F \mid K$ if for very $n \in \mathbb{N}$, any $a_{1}, \ldots, a_{n} \in L$ algebraically independent over $K$ will also be algebraically independent over $F$. Hence, $L \mid K$ is algebraically disjoint from $F \mid K$ if every finitely generated subextension $E \mid K$ of $L \mid K$ satisfies $\operatorname{trdeg} E \mid K=\operatorname{trdeg}$ $E . F \mid F$. As in the case of linear disjointness, the definition of algebraic disjointness is symmetrical. Thus if $L \mid K$ is algebraically disjoint from $F \mid K$ we will also say that $L$ and $F$ are $K$-algebraically disjoint. It is easy to check that if $L \mid K$ is linearly disjoint from $F \mid K$ then it is also algebraically disjoint from $F \mid K$. The converse holds only under additional assumptions. We will use the following case:

Lemma 2.6. Let $L \mid K$ and $F \mid K$ be field extensions algebraically disjoint in a common extension field $\Omega$. If $K$ is relatively algebraically closed in $L$ and $F \mid K$ is separable then $L \mid K$ and $F \mid K$ are also linearly disjoint.

The above properties of linearly disjoint extensions enable us to prove the following lemmas, useful in our constructions.

Lemma 2.7. Take a field $K$ of positive characteristic $p$ and a rational function field $K(x, y) \mid K$. For any $a \in K$ take $L_{a} \mid K\left(y+\frac{a}{x}\right)$ to be a separable algebraic extension such that $K$ is relatively algebraically closed in $L_{a}$. Then for any two distinct elements $a, b \in K$ the extensions $L_{a}(x)$ and $L_{b}(x)$ are linearly disjoint over $K(x, y)$.

Proof. Take two distinct elements $a$ and $b$ of $K$. Since $K\left(y+\frac{a}{x}, y+\frac{b}{x}\right)=K(x, y)$, the elements $y+\frac{a}{x}$ and $y+\frac{b}{x}$ are algebraically independent over $K$. Thus the extensions $K\left(y+\frac{a}{x}\right) \mid K$ and $K\left(y+\frac{b}{x}\right) \mid K$ are algebraically disjoint. Furthermore, $L_{a} \mid K\left(y+\frac{a}{x}\right)$ and $L_{b} \mid K\left(y+\frac{b}{x}\right)$ are algebraic extensions, hence also $L_{a} \mid K$ and $L_{b} \mid K$ are algebraically disjoint. Since $K$ is relatively algebraically closed in $L_{a}$ and $L_{b} \mid K$ is a separable extension, Lemma 2.6 implies that $L_{a}$ and $L_{b}$ are $K$-linearly disjoint.

Applying Lemma 2.1 to the tower $K \subseteq K\left(y+\frac{a}{x}\right) \subseteq L_{a}$ and the extension $L_{b} \mid K$ we deduce that $L_{a}$ and $L_{b} \cdot K\left(y+\frac{a}{x}\right)=L_{b}(x)$ are linearly disjoint over $K\left(y+\frac{a}{x}\right)$. Again, since $K\left(y+\frac{a}{x}\right) \subseteq K(x, y) \subseteq L_{b}(x)$, from the same lemma it follows that $L_{b}(x)$ and $L_{a} . K(x, y)=L_{a}(x)$ are linearly disjoint over $K(x, y)$.

Lemma 2.8. Take a field $K$ of characteristic $p>0$ and a rational function field $K(x, y) \mid K$. For any nonnegative integer $r$ take $L_{r} \mid K\left(\frac{y}{x^{r}}\right)$ to be a (possibly infinite) tower of Artin-Schreier extensions such that $K$ is relatively algebraically closed in $L_{r}$. Then for every two distinct nonnegative integers $r, s$ the extensions $L_{r}(x) \mid K(x, y)$ and $L_{s}(x) \mid K(x, y)$ are linearly disjoint.

Proof. Take $r$ and $s$ to be two distinct nonnegative integers. Without loss of generality we may assume that $t:=r-s>0$. Elements $\frac{y}{x^{s}}$ and $\frac{y}{x^{s}}$ are algebraically independent over $K$, thus the extensions $K\left(\frac{y}{x^{s}}\right)\left|K, K\left(\frac{y}{x^{r}}\right)\right| K$ are algebraically disjoint. Since moreover $L_{r} \mid K\left(\frac{y}{x^{r}}\right)$ and $L_{s} \mid K\left(\frac{y}{x^{s}}\right)$ are algebraic extensions, $K$ is relatively 
algebraically closed in $L_{r}$ and $L_{s} \mid K$ is separable, we can deduce as in the proof of the previous lemma that $L_{r} \mid K$ and $L_{s} \mid K$ are linearly disjoint.

Applying Lemma 2.1 to the tower $K \subseteq K\left(\frac{y}{x^{s}}\right) \subseteq L_{s}$ and the extension $L_{r} \mid K$ we obtain that $L_{s}$ and $L_{r} \cdot K\left(\frac{y}{x^{s}}\right)=L_{r}\left(x^{t}\right)$ are linearly disjoint over $K\left(\frac{y}{x^{s}}\right)$. Using again Lemma 2.1 for the tower $K\left(\frac{y}{x^{s}}\right) \subseteq K\left(\frac{y}{x^{r}}, \frac{y}{x^{s}}\right) \subseteq L_{r}\left(x^{t}\right)$ and the extension $L_{s} \mid K\left(\frac{y}{x^{s}}\right)$ we deduce that $L_{r}\left(x^{t}\right)$ and $L_{s} \cdot K\left(\frac{y}{x^{r}}, \frac{y}{x^{s}}\right)=L_{s}\left(x^{t}\right)$ are linearly disjoint over $K\left(\frac{y}{x^{r}}, \frac{y}{x^{s}}\right)=K\left(\frac{y}{x^{r}}, x^{t}\right)$.

We show that the extensions $L_{s}\left(x^{t}\right)$ and $K(x, y)$ are linearly disjoint over $K\left(\frac{y}{x^{s}}, x^{t}\right)$. By assumption, $L_{s}=\bigcup_{i \in I} L_{s, i}$, where $L_{s, i} \mid L_{s, i-1}$ is a nontrivial Artin-Schreier extension for every $i \in I, L_{s, 0}:=K\left(\frac{y}{x^{s}}\right)$ and $I=\{0, \ldots, n\}$ for some natural number $n$ or $I=\mathbb{N}$. We prove, by induction on $i$, that each of the the extensions $L_{s, i}^{\prime}:=L_{s, i}\left(x^{t}\right)$ is linearly disjoint from $K(x, y)$ over $K\left(\frac{y}{x^{s}}, x^{t}\right)$. Write $t=p^{k} l$, where $k$ is a nonnegative integer and $l \in \mathbb{N}$ is coprime with $p$. Since $x^{t}$ is transcendental over $L_{s}$, also $L_{s, i}\left(x^{t}\right) \mid L_{s, i-1}\left(x^{t}\right)$ is a nontrivial Artin-Schreier extension for very $i \in I$. In the case of $i=1, L_{s, 1}^{\prime} \mid K\left(\frac{y}{x^{s}}, x^{t}\right)$ is an Arin-Schreier extension, hence Galois extension of degree $p$. Suppose that $L_{s, 1}^{\prime}$ and $K(x, y)$ were not linearly disjoint over $K\left(\frac{y}{x^{s}}, x^{t}\right)$. Then by Lemma 2.2 , there would exist $a \in L_{s, 1}^{\prime} \cap K(x, y)$ such that $a \notin K\left(\frac{y}{x^{s}}, x^{t}\right)$. Since $a \in L_{s, 1}^{\prime} \backslash K\left(\frac{y}{x^{s}}, x^{t}\right)$, we would have $K\left(\frac{y}{x^{s}}, x^{t}, a\right)=L_{s, 1}^{\prime}$. On the other hand $a \in K(x, y)$, hence $K(x, y) \mid K\left(\frac{y}{x^{s}}, x^{t}\right)$ would contain a separable subextension of degree $p$. But $K(x, y)=K\left(\frac{y}{x^{s}}, x^{t}\right)(x)$ is a radical extension of degree $t$ of the field $K\left(\frac{y}{x^{s}}, x^{t}\right)$ and the separable degree of the extension is equal to $l$, which is not divisible by $p$, a contradiction.

Take $i \in I, i \geq 1$ and assume that $L_{s, i}^{\prime}$ and $K(x, y)$ are linearly disjoint over $K\left(\frac{y}{x^{s}}, x^{t}\right)$. Hence, in particular, $L_{s, i}^{\prime} \cdot K(x, y)=L_{s, i}^{\prime}(x)$ is an extension of $L_{s, i}^{\prime}$ of degree $t$. Suppose that $L_{s, i+1}^{\prime}$ and $K(x, y)$ were not linearly disjoint over $K\left(\frac{y}{x^{s}}, x^{t}\right)$. Then by Lemma 2.1 , also the extensions $L_{s, i+1}^{\prime}$ and $L_{s, i}^{\prime} \cdot K(x, y)$ would not be linearly disjoint over $L_{s, i}^{\prime}$. However, $L_{s, i}^{\prime} . K(x, y)$ is a radical extension of $L_{s, i}^{\prime}$ of degree $t$ and $L_{s, i+1}^{\prime} \mid L_{s, i}^{\prime}$ is a nontrivial Artin-Schreier extension. The same argument as in the case of $i=1$ leads to a contradiction.

Therefore, $L_{s}\left(x^{t}\right)$ is linearly disjoint from $K(x, y)$ over $K\left(\frac{y}{x^{s}}, x^{t}\right)$. It follows that $L_{s}(x) \mid L_{s}\left(x^{t}\right)$ is a radical extension of degree $t$. Since $L_{r}\left(x^{t}\right) \mid K\left(\frac{y}{x^{s}}, x^{t}\right)$ and $L_{s}\left(x^{t}\right) \mid K\left(\frac{y}{x^{s}}, x^{t}\right)$ are linearly disjoint, $L_{s}\left(x^{t}\right) \cdot L_{r}\left(x^{t}\right)=L_{s}\left(x^{t}\right) \cdot L_{r}$ is a separablealgebraic extension of $L_{s}\left(x^{t}\right)$, being a tower of Artin-Schreier extensions. Repeating the above reasoning we deduce that the extensions $L_{s}(x)$ and $L_{s}\left(x^{t}\right) \cdot L_{r}\left(x^{t}\right)$ are linearly disjoint over $L_{s}\left(x^{t}\right)$. As we have shown, the extensions $L_{r}\left(x^{t}\right)$ and $L_{s}\left(x^{t}\right)$ are linearly disjoint over $K\left(\frac{y}{x^{r}}, x^{t}\right)$. Thus from Lemma 2.1 it follows that also $L_{r}\left(x^{t}\right)$ and $L_{s}(x)$ are linearly disjoint over $K\left(\frac{y}{x^{r}}, x^{t}\right)$.

Finally, applying Lemma 2.1 to the tower $K\left(\frac{y}{x^{s}}, x^{t}\right) \subseteq K(x, y) \subseteq L_{s}(x)$ and the extension $L_{r}\left(x^{t}\right) \mid K\left(\frac{y}{x^{s}}, x^{t}\right)$, we obtain that $L_{s}(x)$ and $L_{r}\left(x^{t}\right) . K(x, y)=L_{r}(x)$ are linearly disjoint over $K(x, y)$.

\subsection{Defect and immediate extensions.}

Take finite field extensions $L \mid K$ and $M \mid L$. Since degree of field extension, ramification index and inertia degree are multiplicative, also the defect is multiplicative. Namely, if a valuation $v$ of $K$ has a unique extension to the field $M$, then

$$
d(M \mid K, v)=d(M \mid L, v) \cdot d(L \mid K, v) .
$$


Note that for a finite extension $(L \mid K, v)$ of valued fields the defect of the extension is equal to its degree if and only if $(L \mid K, v)$ is an immediate extension and it is linearly disjoint from some henselization of $(K, v)$. An infinite extension of valued fields is immediate if and only if every finite subextension of the extension is immediate.

If $(L \mid K, v)$ is an extension of valued fields such that for every element $a \in L$ and $\alpha \in v L$ there is $c \in K$ such that $v(a-c)>\alpha$ then we say that $(K, v)$ is dense in $(L, v)$. If this holds, the extension $(L \mid K, v)$ is immediate. A maximal extension in which $(K, v)$ is dense is unique up to valuation preserving isomorphism over $K$ and called the completion of $(K, v)$. We will denote it by $(K, v)^{c}$, or $K^{c}$ if $v$ is fixed. For a finite extension $F$ of $K$, the valued field $\left(F . K^{c}, v\right)$ is the completion of $(F, v)$, hence we can write $F^{c}=F . K^{c}$. If $v$ is a valuation of rank one, then the completion of $K$ is henselian, hence contains a henselization of $K$.

Take a valued field $(K, v)$. For a valuation $v$ of any rank, $K^{h} \mid K$ is an immediate extension. Fix the extension of $v$ to $\tilde{K}$ and take any algebraic extension $L$ of $K$. Then $L^{h}$ must contain $K^{h}$ and $L$, hence $K^{h} . L \subseteq L^{h}$. Conversely, since every algebraic extension of a henselian field is henselian, $K^{h} \cdot L$ contains $L^{h}$. Therefore $L^{h}=K^{h} . L$.

By Theorem 1 of [Ka], every element $a$ of an immediate extension $(L \mid K, v)$ which does not lie in $K$ is a pseudo limit of a pseudo Cauchy sequence in $(K, v)$ without a limit in $K$. Take a pseudo Cauchy sequence $\left(a_{\nu}\right)_{\nu<\lambda}$ (where $\lambda$ is a limit ordinal) of elements of $K$. Recall that $\left(a_{\nu}\right)_{\nu<\lambda}$ is of transcendental type if for every $g \in K[X]$ the sequence fixes the value of $g$, i.e., there is $\nu_{0}<\lambda$ such that

$$
v g\left(a_{\nu}\right)=v g\left(a_{\nu_{0}}\right) \text { for all } \nu \geq \nu_{0}, \nu<\lambda .
$$

Otherwise, the sequence $\left(a_{\nu}\right)_{\nu<\lambda}$ is of algebraic type. In our constructions we will use the following facts about extensions generated by pseudo limits of pseudo Cauchy sequences (cf. Theorem 2, Theorem 3 of $[\mathrm{Ka}]$ ):

Theorem 2.9. Take a valued field $(K, v)$ and an element $x$ in some valued field extension of $K$. If $x$ is a pseudo limit of a pseudo Cauchy sequence in $(K, v)$ of transcendental type, then $(K(x) \mid K, v)$ is an immediate transcendental extension.

Theorem 2.10. Take a pseudo Cauchy sequence $\left(a_{\nu}\right)_{\nu<\lambda}$ in $(K, v)$ of algebraic type and a polynomial $f$ of minimal degree such that $\left(a_{\nu}\right)_{\nu<\lambda}$ does not fix the value of $f$. If a is a root of $f$, then there is an extension of $v$ to $K(a)$ such that $(K(a) \mid K, v)$ is an immediate extension and $a$ is a pseudo limit of $\left(a_{\nu}\right)_{\nu<\lambda}$.

The following is a special case of Lemma 3.7 of [Ku1]:

Lemma 2.11. Take an extension $(K(x) \mid K, v)$ of valued fields and choose any extension of $v$ to $\widetilde{K(x)}$. Take $K^{h}$ and $K(x)^{h}$ to be the henselizations of $K$ and $K(x)$ respectively in $(\widehat{K(x)}, v)$. If the element $x$ is a pseudo limit of a pseudo Cauchy sequence in $(K, v)$ of transcendental type, then $K^{h}$ is relatively algebraically closed in $K(x)^{h}$.

\subsection{Cuts and distances.}

We will recall only basic notions and facts connected with cuts of ordered abelian groups and distances of elements of valued field extensions. For the details and proofs see Section 2.3 of [Ku3] and Section 3 of $[\mathrm{Ku}-\mathrm{V}]$.

Take a totally ordered set $(T,<)$. For a nonempty subset $S$ of $T$ and an element $a \in T$ we will write $S<a$ if $s<a$ for every $s \in S$. A set $\Lambda^{L} \subseteq T$ is called an initial 
segment of $T$ if for each $\alpha \in \Lambda^{L}$ every $\beta<\alpha$ also lies in $\Lambda^{L}$. A pair $\left(\Lambda^{L}, \Lambda^{R}\right)$ of subsets of $T$ is called a cut in $T$ if $\Lambda^{L}$ is an initial segment of $T$ and $\Lambda^{R}=T \backslash \Lambda^{L}$. To compare cuts in $(T,<)$ we will use the lower cut sets comparison. That is, for two cuts $\Lambda_{1}=\left(\Lambda_{1}^{L}, \Lambda_{1}^{R}\right), \Lambda_{2}=\left(\Lambda_{2}^{L}, \Lambda_{2}^{R}\right)$ in $T$ we will write $\Lambda_{1}<\Lambda_{2}$ if $\Lambda_{1}^{L} \varsubsetneqq \Lambda_{2}^{L}$, and $\Lambda_{1} \leq \Lambda_{2}$ if $\Lambda_{1}^{L} \subseteq \Lambda_{2}^{L}$.

For any $s \in T$ define

$$
\begin{aligned}
& s^{-}:=(\{t \in T \mid t<s\},\{t \in T \mid t \geq s\}), \\
& s^{+}:=(\{t \in T \mid t \leq s\},\{t \in T \mid t>s\}) .
\end{aligned}
$$

We identify the element $s$ with $s^{+}$. Therefore, for a cut $\Lambda=\left(\Lambda^{L}, \Lambda^{R}\right)$ in $T$ and an element $s \in T$ the inequality $\Lambda<s$ means that for every element $\beta \in \Lambda^{L}$ we have $\beta<s$. Similarly, for any subset $M$ of $T$ we define $M^{+}$to be a cut $\left(\Lambda^{L}, \Lambda^{R}\right)$ in $T$ such that $\Lambda^{L}$ is the least initial segment containing $M$, that is,

$$
M^{+}=(\{t \in T \mid \exists m \in M t \leq m\},\{t \in T \mid t>M\}) .
$$

We denote also by $M^{-}$the cut $\left(\Lambda^{L}, \Lambda^{R}\right)$ in $T$ such that $\Lambda^{L}$ is the largest initial segment disjoint with $M$, i.e.,

$$
M^{-}=(\{t \in T \mid t<M\},\{t \in T \mid \exists m \in M t \geq m\}) .
$$

For every extension $(L \mid K, v)$ of valued fields and $z \in L$ define

$$
v(z-K):=\{v(z-c) \mid c \in K\} .
$$

The set $v(z-K) \cap v K$ is an initial segment of $v K$ and thus the lower cut set of a cut in $v K$. However, it is more convenient to work with the cut

$$
\operatorname{dist}(z, K):=(v(z-K) \cap v K)^{+} \text {in the divisible hull } \widetilde{v K} \text { of } v K \text {. }
$$

We call this cut the distance of $z$ from $K$. The lower cut set of dist $(z, K)$ is the smallest initial segment of $\widetilde{v K}$ containing $v(z-K) \cap v K$. If $(F \mid K, v)$ is an algebraic subextension of $(L \mid K, v)$ then $\widetilde{v F}=\widetilde{v K}$. Thus $\operatorname{dist}(z, K)$ and $\operatorname{dist}(z, F)$ are cuts in the same group and we can compare these cuts by set inclusion of the lower cut sets. Since $v(z-K) \subseteq v(z-F)$ we deduce that

$$
\operatorname{dist}(z, K) \leq \operatorname{dist}(z, F) \text {. }
$$

If dist $(z, K)=\left(\Lambda^{L}, \Lambda^{R}\right)$, then for any natural number $n$ we denote by $n$ dist $(z, K)$ the cut in $\widetilde{v K}$ with the lower cut set

$$
n \Lambda^{L}:=\left\{n \gamma \mid \gamma \in \Lambda^{L}\right\} .
$$

Since dist $(z, K)$ is a cut in the divisible group $\widetilde{v K}$, the set $n \Lambda^{L}$ is again an initial segment of $\widetilde{v K}$. We say that the distance $\operatorname{dist}(z, K)$ is idempotent if

$$
n \operatorname{dist}(z, K)=\operatorname{dist}(z, K)
$$

for some natural number $n \geq 2$. From Lemma 2.14 of [Ku3] it follows that this condition holds if and only if dist $(z, K)=H^{+}$or dist $(z, K)=H^{-}$for some convex subgroup $H$ of $\widetilde{v K}$.

If $y$ is another element of $L$ then we define $z \sim_{K} y$ to mean that

$$
v(z-y)>\operatorname{dist}(z, K) \text {. }
$$

If this holds, then $v(z-c)=v(y-c)$ for all $c \in K$ such that $v(z-c) \in v K$ and thus, dist $(z, K)=\operatorname{dist}(y, K)$. The next lemma was proven by Kuhlmann in [Ku3]. It shows that the converse holds under an additional assumption. 
Lemma 2.12. Take a valued field extension $(L \mid K, v)$ and elements $z, y \in L$. If $v(z-K) \cap v K$ has no maximal element then $z \sim_{K} y$ if and only if $v(z-c)=v(y-c)$ for every $c \in K$ such that $v(z-c) \in K$.

The following theorem enables us to deduce an important information about the distance of an Artin-Schreier generator $\vartheta$ of a defect extension $K(\vartheta) \mid K$ from the field $K$ (see Section 2.4).

Theorem 2.13. If $(L \mid K, v)$ is an immediate extension of valued fields, then for every element $z \in L \backslash K$ it follows that $v(z-K) \subseteq v K$ and that $v(z-K)$ has no maximal element. In particular, $v z<\operatorname{dist}(z, K)$.

Using the next property of the relation $\sim_{K}$ one can show that the dependent Artin-Schreier defect extensions can be derived from immediate purely inseparable extensions of degree $p$ (cf. Theorem 2.21 and Section 5). The theorem was proven by Kuhlmann in [Ku4].

Theorem 2.14. Take a valued field $(K, v)$ and the henselization $K^{h}$ of $K$ with respect to some extension of the valuation $v$ to $\tilde{K}$. Assume that $a, z \in \tilde{K}$ are such that

$$
a \sim_{K} z
$$

If a lies in $K^{h}$, then the extensions $K(z)$ and $K^{h}$ are not linearly disjoint over $K$. In particular, the extension $K(z) \mid K$ is not purely inseparable.

For any $\alpha \in v K$ and each cut $\Lambda$ in $v K$ we set $\alpha+\Lambda:=\left(\alpha+\Lambda^{L}, \alpha+\Lambda^{R}\right)$. An immediate consequence of the above definitions is the following lemma:

Lemma 2.15. Take an extension $(L \mid K, v)$ of valued fields. Then for every element $c \in K$ and $y, z \in L$,

a) $\operatorname{dist}(z+c, K)=\operatorname{dist}(z, K)$,

b) $\operatorname{dist}(c z, K)=v c+\operatorname{dist}(z, K)$,

c) if $z \sim_{K} y$, then $z+c \sim_{K} y+c$,

d) if $c \neq 0$ and $z \sim_{K} y$, then $c z \sim_{K} c y$.

\subsection{Artin-Schreier defect extensions.}

We recall a few facts about Artin-Schreier defect extensions of valued fields and their classification presented in detail in [Ku3]. Throughout this section we assume that $(K, v)$ is a valued field of positive characteristic $p$ and $K(\vartheta) \mid K$ an Artin-Schreier extension of degree $p$ with $\vartheta^{p}-\vartheta-a=0$ for some $a \in K$.

Lemma 2.16. If $v a \leq 0$, then $v \vartheta=\frac{1}{p} v a$. If $v a>0$, then exactly one of the conjugates $\vartheta, \vartheta+1, \ldots, \vartheta+p-1$ has value va and the others have value 0 .

We will frequently use the following consequence of Hensel's Lemma:

Lemma 2.17. Assume that $v a>0$ or that $v a=0$ and the polynomial $X^{p}-X-a v$ has root in $K v$. Then the Artin-Schreier generator $\vartheta$ lies in the henselization of $K$ with respect to every extension of $v$ to $\tilde{K}$ and the valuation $v$ of $K$ has exactly $p$ many distinct extensions to $K(\vartheta)$. Therefore equality holds in the fundamental inequality. If $v a=0$ and $X^{p}-X-a v$ has no root in $K v$, then the residue field extension $K(\vartheta) v \mid K v$ is a separable extension of degree $p$. Hence $(K(\vartheta) \mid K, v)$ is defectless.

If $(K(\vartheta) \mid K, v)$ is a defect extension, then $v a<0$. 
Take a field $L$. A polynomial $g \in L[X]$ is called additive if $g(b+c)=g(b)+g(c)$ for all $b, c$ in every extension field $L^{\prime}$ of $L$. Since char $K=p$, the Artin-Schreier polynomial $X^{p}-X$ is additive. Hence for any $c \in K$ the element $\vartheta-c$ is a root of the Artin-Schreier polynomial $X^{p}-X-a+c^{p}-c$. It follows that this polynomial induces the same extension $K(\vartheta) \mid K$ as the polynomial $X^{p}-X-a$. Using this fact one can easily show a consequence of the above lemma:

Corollary 2.18. If $(K(\vartheta) \mid K, v)$ has nontrivial defect, then $v(\vartheta-c)<0$ for every $c \in K$ and consequently $\operatorname{dist}(\vartheta-K) \leq 0^{-}$.

Assume that $(K(\vartheta) \mid K, v)$ is a defect extension. Then the defect is equal to the degree of the extension, hence $(K(\vartheta) \mid K, v)$ is immediate. By Theorem 2.13 it follows that $v(\vartheta-K) \cap v K=v(\vartheta-K)$, and that $v(\vartheta-K)$ has no maximal element.

Take $\vartheta^{\prime} \in K(\vartheta)$ to be another Artin-Schreier generator of the extension $K(\vartheta) \mid K$. One can show that the element $\vartheta^{\prime}$ is of the form $i \vartheta+c$ for some $i \in \mathbb{F}_{p}^{\times}$and $c \in K$ (cf. Lemma 2.26 of [Ku3]). Hence from Lemma 2.15 it follows that $\delta:=\operatorname{dist}(\vartheta, K)$ does not depend on the choice of the Artin-Schreier generator. We call $\delta$ the distance of the Artin-Schreier extension $(K(\vartheta) \mid K, v)$. Corollary 2.18 implies that $\delta \leq 0^{-}$.

In Section 1, we have introduced a classification of Artin-Schreier defect extensions into "dependent" and "independent" ones. If $(K(\vartheta) \mid K, v)$ is a defect extension, then from Lemma 2.12 it follows that $(K(\vartheta) \mid K, v)$ is a dependent ArtinSchreier defect extension if and only if there is an immediate purely inseparable extension $K(\eta) \mid K$ of degree $p$ such that

$$
\eta \sim_{k} \vartheta
$$

The next proposition gives us a useful characterization of independent Artin-Schreier defect extension by idempotent cuts.

Proposition 2.19. Assume that the extension $(K(\vartheta) \mid K, v)$ has nontrivial defect. Then $K(\vartheta) \mid K$ is an independent Artin-Schreier defect extension if and only if its distance is idempotent.

If $\delta$ is idempotent, then $\delta=H^{+}$or $\delta=H^{-}$for some convex subgroup $H$ of $\widetilde{v K}$. If moreover $K(\vartheta) \mid K$ is a defect extension, then $\delta \leq 0^{-}$, hence $\delta=H^{-}$. In particular, if the value group of $(K, v)$ is archimedean, then the unique proper convex subgroup of $\widetilde{v K}$ is $\{0\}$.

Corollary 2.20. Assume that $(K(\vartheta) \mid K, v)$ has nontrivial defect and the valuation $v$ is of rank one. Then the Artin-Schreier defect extension $(K(\vartheta) \mid K, v)$ is independent if and only if $\operatorname{dist}(\vartheta, K)=0^{-}$

Suppose that the field $K$ admits an immediate purely inseparable extension $K(\eta) \mid K$ of degree $p$ such that $\eta \in K^{1 / p} \backslash K^{c}$. For any element $b \in K$ consider the polynomial

$$
f_{b}=Y^{p}-b^{p-1} Y-\eta^{p} .
$$

With each of the polynomials $f_{b}$ we can associate through the transformation $Y=b X$ the Artin-Schreier polynomial

$$
g_{b}(X)=X^{p}-X-\left(\frac{\eta}{b}\right)^{p}
$$

Note that $\vartheta_{b}$ is a root of the polynomial $g_{p}$ if and only if $b \vartheta_{b}$ is a root of $f_{b}$. The next theorem shows when such deformation of a polynomial $Y^{p}-\eta^{p}$ generating a purely 
inseparable extension into an Artin-Schreier polynomial leads to a dependent ArtinSchreier defect extension.

Theorem 2.21. Suppose that the polynomial $X^{p}-\eta^{p} \in K[X]$ induces an immediate extension of $(K, v)$ which does not lie in the completion of $K$. Then for each $b \in K^{\times}$ such that

$$
(p-1) v b>p \operatorname{dist}(\eta, K)-v \eta
$$

the polynomial $g_{b}=X^{p}-X-\left(\frac{\eta}{b}\right)^{p}$ induces a dependent Artin-Schreier defect extension. Moreover, every root $\vartheta_{b}$ of $g_{b}$ satisfies

$$
\vartheta_{b} \sim_{K} \frac{\eta}{b}
$$

\section{Dependent Artin-Schreier Defect extensions of VAlued fields}

In this section we prove Theorem 1.4. We show that under the assumptions of the theorem the valued field $(K, v)$ admits infinitely many parallel dependent ArtinSchreier defect extensions, such that the compositum of any $n$ of the extensions is a Galois extension of degree and defect $p^{n}$. Throughout this section we assume that $(K, v)$ is a valued field of positive characteristic $p$ with perfect residue field and $p$-divisible value group.

Proof of Theorem 1.4: Suppose there is an element $a \in K$ such that $a^{1 / p^{n}}$ does not lie in the completion $K^{c}$ of $K$. Take

$$
k:=\min \left\{i \in \mathbb{N} \mid a^{1 / p^{i}} \notin K^{c}\right\} .
$$

Since $a^{1 / p^{k}} \notin K^{c}$, there is $\gamma \in v K$ such that $v\left(a^{1 / p^{k}}-K\right)<\gamma$. By definition of $k$, the element $a^{1 / p^{k-1}}$ lies in the completion of $K$. Hence, $v\left(a^{1 / p^{k-1}}-d\right)>p \gamma$ for some $d \in K$. Thus

$$
v\left(a^{1 / p^{k}}-K\right)<\gamma<v\left(a^{1 / p^{k}}-d^{1 / p}\right) .
$$

It follows that also $v\left(d^{1 / p}-K\right)<\gamma$ and consequently $d^{1 / p} \notin K^{c}$.

Since the value group of the field $K$ is $p$-divisible and its residue field is perfect, $d^{1 / p}$ generates an immediate purely inseparable extension of $K$ which does not lie in the completion of $K$. By Theorem 2.21, we can choose an element $b_{1} \in K^{\times}$of large enough value, such that a root $\vartheta_{1}$ of the polynomial

$$
f_{1}=Y^{p}-Y-\frac{d}{b_{1}^{p}}
$$

generates a dependent Artin-Schreier defect extension $K_{1}=K_{0}\left(\vartheta_{1}\right)$ of $K_{0}:=K$.

Take a natural number $n$ and assume that we have chosen $K_{1}, \ldots, K_{n}$ to be algebraic extensions of $K$ such that each $K_{i}=K_{i-1}\left(\vartheta_{i}\right)$ is a dependent ArtinSchreier defect extension of $K_{i-1}$, where $\vartheta_{i}$ is a root of the polynomial

$$
f_{i}=Y^{p}-Y-\frac{1}{b_{i}^{p}} \vartheta_{i-1}
$$

for some $b_{i} \in K^{\times}$. Assume in addition that $\vartheta_{i}^{1 / p} \notin K_{i}^{c}$ for every $i \leq n-1$. Note that then also $\vartheta_{n}^{1 / p}$ does not lie in the completion of $K_{n}$. Indeed, suppose that $\vartheta_{n}^{1 / p} \in K_{n}^{c}=K_{n-1}^{c}\left(\vartheta_{n}\right)$. Since $\vartheta_{n}^{p}-\vartheta_{n}=\frac{1}{b_{n}^{p}} \vartheta_{n-1}$, we have that $\vartheta_{n}-\vartheta_{n}^{1 / p}=\frac{1}{b_{n}} \vartheta_{n-1}^{1 / p}$. Therefore,

$$
\vartheta_{n-1}^{1 / p}=b_{n}\left(\vartheta_{n}-\vartheta_{n}^{1 / p}\right) \in K_{n-1}^{c}\left(\vartheta_{n}\right)
$$


By assumption $\vartheta_{n-1}^{1 / p} \notin K_{n-1}^{c}$, thus we would obtain that $K_{n-1}^{c}\left(\vartheta_{n-1}^{1 / p}\right)=K_{n-1}^{c}\left(\vartheta_{n}\right)$, but $K_{n-1}^{c}\left(\vartheta_{n-1}^{1 / p}\right)$ is a nontrivial purely inseparable and $K_{n-1}^{c}\left(\vartheta_{n}\right)$ a separable extension of $K_{n-1}^{c}$, a contradiction.

Then, using the same argument as before, we can choose an element $b_{n+1} \in K^{\times}$ such that the polynomial

$$
f_{n+1}=Y^{p}-Y-\frac{1}{b_{n+1}^{p}} \vartheta_{n}
$$

induces a dependent Artin-Schreier defect extension $K_{n}\left(\vartheta_{n+1}\right) \mid K_{n}$, where $\vartheta_{n+1}$ is a root of the polynomial $f_{n+1}$. By induction we obtain an infinite chain $K_{n} \mid K_{n-1}$ of dependent Artin-Schreier defect extensions.

Assume now that every purely inseparable extension of $(K, v)$ lies in the completion of $K$. Suppose there were a dependent Artin-Schreier defect extension $(K(\vartheta) \mid K, v)$. Then there would be $\eta \in K^{1 / p}$ such that $\eta \sim_{K} \vartheta$, that is,

$$
v(\vartheta-\eta)>v(\eta-K) \text {. }
$$

Since $\eta \in K^{1 / p} \subseteq K^{c}$ we would obtain $\eta=\vartheta$, a contradiction.

An immediate consequence of the theorem is the following:

Corollary 3.1. If $(K, v)$ admits at least one dependent Artin-Schreier defect extension, then it admits an infinite tower of such extensions.

Proof. Assume that $(K, v)$ admits a dependent Artin-Schreier defect extension $(K(\vartheta) \mid K, v)$. Then, from the definition of dependent extension, $K$ admits a purely inseparable extension $K(\eta)$ of degree $p$ such that $\vartheta \sim_{K} \eta$. From the proof of the above theorem if follows that $\eta$ does not lie in the completion of the field $K$. Therefore, from Theorem 1.4 we obtain that $K$ admits an infinite tower of dependent Artin-Schreier defect extensions.

We modify now the above construction to obtain infinitely many parallel dependent Artin-Schreier defect extensions of the field $K$. Set $L_{0}:=K$. As before, we choose an element $d \in K$ whose $p$-th root does not lie in the completion of $K$ and take $c_{1} \in K^{\times}$such that a root $\eta_{1}$ of the polynomial

$$
h_{1}=Y^{p}-Y-\frac{d}{c_{1}^{p}}
$$

generates a dependent Artin-Schreier defect extension $L_{1}=L_{0}\left(\eta_{1}\right)$ of $L_{0}:=K$. Take $n \in \mathbb{N}$ and assume that we have chosen $L_{1}, \ldots, L_{n}$ to be algebraic extensions of $K$ such that $L_{i}=L_{i-1}\left(\eta_{i}\right)$ is a dependent Artin-Schreier defect extension of $L_{i-1}$ generated by a root $\eta_{i}$ of the polynomial

$$
h_{i}=Y^{p}-Y-\frac{d}{c_{i}^{p}}
$$

for some $c_{i} \in K^{\times}$. Assume in addition that $d^{1 / p} \notin L_{i}^{c}$ for every $i \leq n-1$. Suppose that $d^{1 / p} \in L_{n}^{c}=L_{n-1}^{c}\left(\eta_{n}\right)$. Since $d^{1 / p}$ does not lie in the completion of $L_{n-1}$, we have that $\left[L_{n-1}^{c}\left(d^{1 / p}\right): L_{n-1}^{c}\right]=p$. Therefore we would obtain $L_{n-1}^{c}\left(d^{1 / p}\right)=L_{n-1}^{c}\left(\eta_{n}\right)$, but $L_{n-1}^{c}\left(d^{1 / p}\right)$ is a nontrivial purely inseparable extension of $L_{n-1}^{c}$ and the extension $L_{n-1}^{c}\left(\eta_{n}\right) \mid L_{n-1}^{c}$ is separable, a contradiction. Consequently $d^{1 / p} \notin L_{n}^{c}$. By Theorem 2.21 , we can choose an element $c_{n+1} \in K^{\times}$of 
large enough value, such that a root $\eta_{n+1}$ of the polynomial

$$
h_{n+1}=Y^{p}-Y-\frac{d}{c_{n+1}^{p}}
$$

generates a dependent Artin-Schreier defect extension $L_{n+1}=L_{n}\left(\eta_{n+1}\right)$ of $L_{n}$. Hence we obtain an infinite chain of dependent Artin-Schreier defect extensions $L_{n} \mid L_{n-1}$.

Take a natural number $n$. Since every polynomial $h_{n}$ has coefficients in $K$, the field $K\left(\eta_{n}\right)$ is a nontrivial Artin-Schreier extension of $K$. By what we have shown, the valuation $v$ of $K$ has a unique extension to the field $L_{n}$ and the extension $L_{n} \mid K$ is immediate. Since $K\left(\eta_{n}\right) \mid K$ is a subextension of $L_{n} \mid K$, we deduce that $v$ has also a unique extension to $K\left(\eta_{n}\right)$ and $K\left(\eta_{n}\right) \mid K$ is immediate. Hence $K\left(\eta_{n}\right) \mid K$ has nontrivial defect. From Proposition 2.21 it follows that $\eta_{n} \sim_{L_{n-1}} \frac{1}{c_{n}} d^{1 / p}$. Thus

$$
v\left(\eta_{n}-\frac{d^{1 / p}}{c_{n}}\right)>v\left(\frac{d^{1 / p}}{c_{n}}-L_{n-1}\right) \geq v\left(\frac{d^{1 / p}}{c_{n}}-K\right) .
$$

It follows that $\eta_{n} \sim_{K} \frac{1}{c_{n}} d^{1 / p}$ and $K\left(\eta_{n}\right) \mid K$ is a dependent Artin-Schreier defect extension. Since for every $n \in \mathbb{N}$ the extension $L_{n} \mid L_{n-1}$ is nontrivial, we deduce that $K$ admits infinitely many dependent Artin-Schreier extensions.

Take $n \in \mathbb{N}$, any distinct natural numbers $i_{1}, \ldots, i_{n}$ and consider the compositum $K\left(\eta_{i_{1}}, \ldots, \eta_{i_{n}}\right)$ of the fields $K\left(\eta_{i_{1}}\right), \ldots, K\left(\eta_{i_{n}}\right)$. Since $K\left(\eta_{i_{1}}, \ldots, \eta_{i_{n}}\right) \mid K$ is a subextension of some $L_{m} \mid K$, we deduce that the valuation $v$ of $K$ has a unique extension to $K\left(\eta_{i_{1}}, \ldots, \eta_{i_{n}}\right)$ and the extension $K\left(\eta_{i_{1}}, \ldots, \eta_{i_{n}}\right) \mid K$ is immediate. Consequently, the defect of the extension is equal to its degree. By what we have proved, $L_{m} \mid K$ is of degree $p^{m}$. From the definition of $L_{m}$ it follows that $K\left(\eta_{i_{1}}, \ldots, \eta_{i_{n}}\right) \mid K$ must be of degree $p^{n}$. Furthermore the extension is Galois, as a compositum of Galois extensions of the field $K$. We have thus proved:

Proposition 3.2. If there is a purely inseparable extension of $(K, v)$ which does not lie in the completion of the field, then $K$ admits infinitely many dependent ArtinSchreier defect extensions such that the compositum of any $n$ of the extensions is a Galois extension of $K$ of degree and defect $p^{n}$.

Corollary 3.3. If $(K, v)$ admits at least one dependent Artin-Schreier defect extension, then it admits infinitely many dependent Artin-Schreier defect extensions such that the compositum of any $n$ of the extensions is a Galois extension of $K$ of degree and defect $p^{n}$.

From the above corollary it follows immediately that if the field $(K, v)$ admits only finitely many Artin-Schreier defect extensions, then all of the extensions are independent.

\section{Constructions of towers of Artin-Schreier Defect extensions of RATIONAL FUNCTION FIELDS.}

This section is devoted to the proofs of Theorems 1.1, 1.2 and 1.3. Throughout the section we will consider the following situation. We assume $K$ to be a field of positive characteristic $p$. We take $(K(x) \mid K, v)$ to be the rational function field equipped with the $x$-adic valuation $v$. We set $v x=1$. The field can be considered as a subfield of the power series field $\left(K\left(\left(x^{\Gamma}\right)\right), v_{x}\right)$ with the canonical valuation $v_{x}$ and a group $\Gamma \subseteq \mathbb{Q}$. 
In the following constructions we choose the element $y \in K\left(\left(x^{\Gamma}\right)\right)$ to be a pseudo limit of a pseudo Cauchy sequence of transcendental type in some subfield of $K\left(\left(x^{\Gamma}\right)\right)$ containing $K(x)$. We consider the field $K(x, y)$ with the restriction $v$ of the $x$-adic valuation $v_{x}$ of $K\left(\left(x^{\Gamma}\right)\right)$. By Theorem 2.9, such element $y$ is transcendental over $K(x)$. Hence $(K(x, y), v)$ can be viewed as a rational function field with valuation $v$ described by its restriction to $K(x)$ and the choice of $y$.

Take $y$ to be a power series

$$
y=\sum_{i=1}^{\infty} x^{n_{i} p^{-e_{i}}} \in K\left(\left(x^{\frac{1}{p^{\infty}} \mathbb{Z}}\right)\right),
$$

where $\left(e_{i}\right)_{i \in \mathbb{N}}$ is a strictly increasing sequence of natural numbers such that

$$
e_{i+1}-e_{i} \geq i
$$

for every $i \in \mathbb{N}$, and $\left(n_{i}\right)_{i \in \mathbb{N}}$ is a sequence of integers coprime with $p$ and such that $\left(n_{i} p^{-e_{i}}\right)_{i \in \mathbb{N}}$ is strictly increasing.

Then $(K(x, y), v)$ is a subfield of $\left(K\left(\left(x^{\frac{1}{p^{\infty}} \mathbb{Z}}\right)\right), v_{x}\right)$ defined by the following conditions:

$$
\left\{\begin{array}{l}
K \text { is a field of characteristic } p>0, \\
K(x) \mid K \text { is the rational function field, } \\
y \in K\left(\left(x^{\frac{1}{p^{\infty}} \mathbb{Z}}\right)\right) \text { is of the form }(2), \\
v \text { is the restriction of the valuation } v_{x} \text { to the field } K(x, y) .
\end{array}\right.
$$

Consequently we obtain that

$$
K(x, y) v \subseteq K\left(\left(x^{\frac{1}{p^{\infty}} \mathbb{Z}}\right)\right) v=K \text { and } v K(x, y) \subseteq v K\left(\left(x^{\frac{1}{p^{\infty}}} \mathbb{Z}\right)\right)=\frac{1}{p^{\infty}} \mathbb{Z} .
$$

Moreover, since $K=K(x) v \subseteq K(x, y) v$, it follows that $K(x, y) v=K$. We show that equality holds also for the value groups of $K(x, y)$ and the power series field. For any natural number $j$ we have that

$$
z_{j}:=\sum_{i=j+1}^{\infty} x^{n_{i} p^{e_{j}-e_{i}}}=y^{p^{e_{j}}}-\sum_{i=1}^{j} x^{n_{i} p^{e_{j}-e_{i}}} \in K(x, y),
$$

by the assumption on $\left(e_{i}\right)_{i \in \mathbb{N}}$. Thus $v z_{j}=n_{j+1} p^{e_{j}-e_{j+1}}$. Since $e_{j}-e_{j+1} \leq-j$, the element $n_{j+1} p^{-j}$ lies in $v K(x, y)$. As $n_{j+1}$ is coprime with $p$, also $p^{-j}$ lies in $v K(x, y)$. Hence $\frac{1}{p^{\infty}} \mathbb{Z} \subseteq v K(x, y)$ and consequently

$$
v K(x, y)=\frac{1}{p^{\infty}} \mathbb{Z}=v_{x} K\left(\left(x^{\frac{1}{p^{\infty}} \mathbb{Z}}\right)\right) .
$$

Therefore in particular, $\left(K\left(\left(x^{\frac{1}{p^{\infty}} \mathbb{Z}}\right)\right) \mid K(x, y), v_{x}\right)$ is an immediate extension.

Consider the subfield $L:=K\left(x^{p^{-i}} \mid i \in \mathbb{N}\right)$ of the power series field. For every natural number $n$, set

$$
a_{n}:=\sum_{i=1}^{n} x^{n_{i} p^{-e_{i}}} \in L .
$$

Then the element $y$ is a pseudo limit of the pseudo Cauchy sequence $\left(a_{n}\right)_{n \in \mathbb{N}}$ in $L$. We show that the sequence is of transcendental type. Suppose the sequence is of algebraic type. Then by Theorem 2.10, there exists an algebraic extension $(L(b) \mid L, v)$ such the element $b$ is a pseudo limit of the sequence. Thus also the 
extension $K(x, b) \mid K(x)$ is algebraic and consequently finite. On the other hand, $\frac{1}{p^{\infty}} \mathbb{Z} \subseteq v K(x, b)$. Indeed, for every $j \in \mathbb{N}$ consider the value of the element

$$
b^{p^{e_{j}}}-a_{j}^{p^{e_{j}}}=b^{p^{e_{j}}}-\sum_{i=1}^{j} x^{n_{i} p^{e_{j}-e_{i}}} \in K(x, b) .
$$

Since $b$ is a pseudo limit of $\left(a_{n}\right)_{n \in \mathbb{N}}$, we have

$$
v\left(b^{p^{e_{j}}}-a_{j}^{p^{e_{j}}}\right)=p^{e_{j}} v\left(a_{j+1}-a_{j}\right)=n_{j+1} p^{e_{j}-e_{j+1}} .
$$

As before we deduce that $p^{-j} \in v K(x, b)$. Therefore, $\frac{1}{p^{\infty}} \mathbb{Z} \subseteq v K(x, b)$ and $(v K(x, b): v K(x))=\infty$, a contradiction to the fundamental inequality. Hence the pseudo Cauchy sequence $\left(a_{n}\right)_{n \in \mathbb{N}}$ is of transcendental type and from Theorem 2.9 it follows that $y$ is transcendental over $K(x)$.

Lemma 4.1. The ring $K\left[x, \frac{1}{x}, y\right]$ is dense in the field $(K(x, y), v)$

Proof. Take any element $u \in K(x, y)$. Choose $f, g \in K\left[x, \frac{1}{x}, y\right]$ such that $u=\frac{f}{g}$. Without loss of generality we may assume that $v g=0$. Indeed, suppose that $v g=\frac{a}{p^{k}}$, where $a, k$ are integers and $k \geq 0, a \neq 0$. Choose $m, d \in \mathbb{Z}$ such that $d \geq 0$ and $\frac{a}{p^{k}}=m+\frac{d}{p^{k}}$. Take $j=e_{k+1}-e_{k}-k \geq 0$ and set

$$
\tilde{w}:=\left(y^{p^{e_{k}}}-\sum_{i=1}^{k} x^{n_{i} p^{e_{k}-e_{i}}}\right)^{p^{j}}=x^{n_{k+1} p^{-k}}+\sum_{i=k+2}^{\infty} x^{n_{i} p^{e_{k}-e_{i}+j}} .
$$

Then $v \tilde{w}=n_{k+1} p^{-k}$. Since by assumption $n_{k+1}$ is coprime with $p$, there are integers $l$ and $t>0$ such that

$$
l p^{k}+t n_{k+1}=-1
$$

Then for $w:=\left(x^{l} \tilde{w}^{t}\right)^{d} \in K\left[x, \frac{1}{x}, y\right]$ we obtain

$$
v w=d\left(l+t n_{k+1} p^{-k}\right)=-\frac{d}{p^{k}} .
$$

Hence $u=\frac{x^{-m} w f}{x^{-m} w g}$, where $v x^{-m} w g=0$.

Therefore, $g$ is of the form

$$
\sum_{q \in \frac{1}{p^{\infty}} \mathbb{Z}, q \geq 0} a_{q} x^{q}
$$

with $a_{q} \in K$ and $a_{0} \neq 0$. Set $\tilde{f}:=a_{0}^{-1} f$ and $h=-a_{0}^{-1}\left(g-a_{0}\right)$. Then $\tilde{f}$ and $h$ are elements of $K\left[x, \frac{1}{x}, y\right]$ such that $v h>0$ and

$$
u=\frac{f}{g}=\frac{\tilde{f}}{1-h} .
$$

Take any $\alpha \in v K(x, y)$. Since $v h>0$, there is a natural number $N$ such that $v \tilde{f}+(N+1) v h>\alpha$. Hence for

$$
u_{N}:=\tilde{f} \sum_{j=0}^{N} h^{j} \in K\left[x, \frac{1}{x}, y\right]
$$


we obtain that

$v\left(u-u_{N}\right)=v\left(\frac{\tilde{f}}{1-h}-\tilde{f} \sum_{j=0}^{N} h^{j}\right)=v \tilde{f}+v\left(\frac{h^{N+1}}{1-h}\right)=v \tilde{f}+(N+1) v h>\alpha$.

This shows that $K\left[x, \frac{1}{x}, y\right]$ is dense in $K(x, y)$.

4.1. Towers of independent and dependent Artin-Schreier defect extensions of rational function fields.

This section is devoted to the proofs of Theorems 1.1, 1.2 and 1.3. Throughout the section we take $(K(x, y), v)$ to be a valued rational function field satisfying the assumptions (3).

To prove Theorem 1.1, we will need the following lemma:

Lemma 4.2. Take an element

$$
u=\sum_{i=m}^{\infty} a_{i} x^{-p^{-i}} \in K\left(\left(x^{\frac{1}{p^{\infty}} \mathbb{Z}}\right)\right),
$$

where $m$ is an integer and the coefficients $a_{i}$ lie in some perfect subfield $E$ of $K$. Then for every natural number $n$ a root $\eta$ of the polynomial $Y^{p^{n}}-Y-u$ can be chosen to be of the form

$$
\eta=\sum_{i=m+n}^{\infty} c_{i} x^{-p^{-i}}
$$

with $c_{i} \in E$.

Proof. One can easily check that the element

$$
\vartheta=\sum_{i=m+n}^{\infty} c_{i} x^{-p^{-i}}
$$

where $c_{i} \in E$ are of the form

$$
\begin{array}{ll}
c_{i}=a_{i-n}^{p^{-n}} & \text { for } i=m+n, \ldots, m+2 n-1, \\
c_{i}=\left(a_{i-n}+c_{i-n}\right)^{p^{-n}} & \text { for } i \geq m+2 n,
\end{array}
$$

satisfies $\vartheta^{p^{n}}-\vartheta=u$.

Now we are able to give the

Proof of Theorem 1.1: Assume that $(K(x, y), v)$ satisfies the assumptions (3) with $n_{i}=-1$ for every $i \in \mathbb{N}$ in (2). Then the element $y$ is of the form

$$
y=\sum_{i=1}^{\infty} x^{-p^{-e_{i}}}
$$

Suppose that $E$ is a perfect subfield of $K$ of cardinality $\kappa$. Take any $a \in E$ and consider the field $\left(K\left(y+\frac{a}{x}\right), v\right)$. Since $v\left(y+\frac{a}{x}\right)=-1$ and the valuation $v$ is trivial

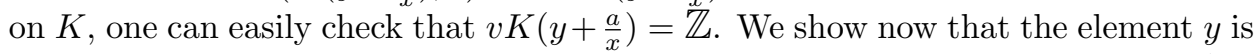
a pseudo limit of a pseudo Cauchy sequence in the perfect hull $F_{a}:=K\left(y+\frac{a}{x}\right)^{1 / p^{\infty}}$ of $K\left(y+\frac{a}{x}\right)$. More precisely, we construct a sequence of elements $b_{k} \in F_{a}$ of the form

$$
b_{k}=y-\sum_{i=m_{k}}^{\infty} a_{i}^{(k)} x^{-p^{-i}}
$$


where $\left(m_{k}\right)_{k \in \mathbb{N}}$ is a strictly increasing sequence of natural numbers and $a_{i}^{(k)} \in K$, $a_{m_{k}}^{(k)} \neq 0$. Then for every $k$ and $l$ such that $k<l$ we obtain $v\left(b_{k}-b_{l}\right)=-p^{-m_{k}}$. Thus $\left(b_{k}\right)_{k \in \mathbb{N}}$ is a pseudo Cauchy sequence. Since

$$
v\left(y-b_{k}\right)=-p^{-m_{k}}=v\left(b_{l}-b_{k}\right)
$$

for $l>k$, the element $y$ is a pseudo limit of the pseudo Cauchy sequence $\left(b_{k}\right)_{k \in \mathbb{N}}$.

We start the construction with

$$
b_{1}:=a^{-p^{-e_{1}}}\left(y+\frac{a}{x}\right)^{p^{-e_{1}}}=x^{-p^{-e_{1}}}+a^{-p^{-e_{1}}} y^{p^{-e_{1}}} .
$$

Then, using the fact that $\left(e_{i}\right)_{i \in \mathbb{N}}$ is a strictly increasing sequence of natural numbers we obtain that

$$
\begin{aligned}
y-b_{1} & =\sum_{i=1}^{\infty} x^{-p^{-e_{i}}}-x^{-p^{-e_{1}}}-a^{-p^{-e_{1}}} \sum_{i=1}^{\infty} x^{-p^{-e_{i}-e_{1}}} \\
& =\sum_{i=2}^{\infty} x^{-p^{-e_{i}}}-a^{-p^{-e_{1}}} \sum_{i=1}^{\infty} x^{-p^{-e_{i}-e_{1}}}=\sum_{i=m_{2}}^{\infty} a_{i}^{(2)} x^{-p^{-i}},
\end{aligned}
$$

where $m_{2} \geq 2$ and $a_{m_{2}}^{(2)} \neq 0$. Assume that we have constructed $b_{1}, \ldots, b_{j} \in F_{a}$ of the form (4) for every $k \leq j$. Set

$b_{j+1}:=b_{j}+a^{-p^{-m_{j}}} a_{m_{j}}^{(j)}\left(y+\frac{a}{x}\right)^{p^{-m_{j}}}=b_{j}+a^{-p^{-m_{j}}} a_{m_{j}}^{(j)} y^{p^{-m_{j}}}+a_{m_{j}}^{(j)} x^{-p^{-m_{j}}} \in F_{a}$.

Then we have

$$
\begin{aligned}
y-b_{j+1} & =y-b_{j}-a^{-p^{-m_{j}}} a_{m_{j}}^{(j)} y^{p^{-m_{j}}}-a_{m_{j}}^{(j)} x^{p^{-m_{j}}} \\
& =\sum_{i=m_{j}+1}^{\infty} a_{i}^{(j)} x^{-p^{-i}}-a^{-p^{-m_{j}}} a_{m_{j}}^{(j)} \sum_{i=1}^{\infty} x^{-p^{-e_{i}-m_{j}}}=\sum_{i=m_{j+1}}^{\infty} a_{i}^{(j+1)} x^{-p^{-i}},
\end{aligned}
$$

for some $a_{i}^{(j+1)} \in K$ and a natural number $m_{j+1}$ such that $a_{m_{j+1}} \neq 0$. Since $e_{1}+m_{j} \geq m_{j}+1$ we have that $m_{j+1}>m_{j}$.

We now use a similar argument as before to show that the pseudo Cauchy sequence $\left(b_{k}\right)_{k \in \mathbb{N}}$ is of transcendental type. Suppose the sequence were of algebraic type. Then, by Theorem 2.10 there would exist an algebraic extension $\left(F_{a}(b) \mid F_{a}, v\right)$ with $b$ a pseudo limit of the sequence. Then the element $b$ would be also algebraic over $K\left(y+\frac{a}{x}\right)$. Thus the extension $K\left(y+\frac{a}{x}, b\right) \mid K\left(y+\frac{a}{x}\right)$ would be finite. On the other hand, $v K\left(y+\frac{a}{x}, b\right)=\frac{1}{p^{\infty}} \mathbb{Z}$. Indeed, for any $j \in \mathbb{N}$ consider the value of the element

$$
\begin{aligned}
u_{j} & :=b^{p^{e_{j}}}-\sum_{i=1}^{j}\left(a^{-1}\left(y+\frac{a}{x}-b\right)\right)^{p^{e_{j}-e_{i}}} \\
& =b^{p^{e_{j}}}-\sum_{i=1}^{j} x^{-p^{e_{j}-e_{i}}}-\sum_{i=1}^{j}\left(a^{-1}(y-b)\right)^{p^{e_{j}-e_{i}}} .
\end{aligned}
$$

Using Lemma 2.5 for the field $L=K\left(y+\frac{a}{x}\right)$ we can extend the valuations of $K\left(y+\frac{a}{x}, b\right)$ and $K(x, y)$ to a valuation of $K(x, y, b)$. Denote this extension again 
by $v$. Then

$$
v u_{j}=v\left((b-y)^{p^{e_{j}}}+y^{p^{e_{j}}}-\sum_{i=1}^{j} x^{-p^{e_{j}-e_{i}}}-\sum_{i=1}^{j}\left(a^{-1}(y-b)\right)^{p^{e_{j}-e_{i}}}\right) .
$$

Since $v\left(b-b_{k}\right)=v\left(y-b_{k}\right)=v\left(b_{k+1}-b_{k}\right)=-p^{-m_{k}}$, by the ultrametric triangle law we have that

$$
v(b-y)=v\left(b-b_{k}+b_{k}-y\right) \geq-p^{-m_{k}} \geq-p^{-k}
$$

for every natural number $k$. Hence $v(b-y) \geq 0$. Moreover,

$$
v\left(y^{p^{e_{j}}}-\sum_{i=1}^{j} x^{-p^{e_{j}-e_{i}}}\right)=v\left(\sum_{i=j+1}^{\infty} x^{-p^{e_{j}-e_{i}}}\right)=-p^{e_{j}-e_{j+1}}
$$

and thus $v u_{j}=-p^{e_{j}-e_{j+1}}$, where $e_{j}-e_{j+1} \leq-j$. It follows that $p^{-j} \in v K\left(y+\frac{a}{x}, b\right)$ and the value group is $p$-divisible. This contradicts the fundamental inequality, since $K\left(y+\frac{a}{x}, b\right) \mid K\left(y+\frac{a}{x}\right)$ was finite and $v\left(K\left(y+\frac{a}{x}\right)=\mathbb{Z}\right.$. Therefore, the pseudo Cauchy sequence $b_{k}$ must be of transcendental type.

Using Lemma 2.11 , we conclude that the field $F_{a}^{h}$ is relatively algebraically closed in $F_{a}(y)^{h}$. Since $K(x, y)^{h} \mid K\left(y+\frac{a}{x}\right)$ is separable and therefore linearly disjoint from $F_{a} \mid K\left(y+\frac{a}{x}\right)$, Lemma 2.1 shows that $K(x, y)^{h}$ and $K\left(y+\frac{a}{x}\right)^{h} \cdot F_{a}=\left(K\left(y+\frac{a}{x}\right)^{h}\right)^{1 / p^{\infty}}$ are linearly disjoint over $K\left(y+\frac{a}{x}\right)^{h}$. Hence the extension $K(x, y)^{h} \mid K\left(y+\frac{a}{x}\right)^{h}$ is separable. We show that from these facts follows that $K\left(y+\frac{a}{x}\right)^{h}$ is relatively algebraically closed in $K(x, y)^{h}$. Suppose there were an element $z \in K(x, y)^{h} \backslash K\left(y+\frac{a}{x}\right)^{h}$ algebraic over $K\left(y+\frac{a}{x}\right)^{h}$. Then $z$ would be separable over $K\left(y+\frac{a}{x}\right)^{h}$. Since $K(x, y)^{h} \subseteq F_{a}(y)^{h}$ and $F_{a}^{h}=K\left(y+\frac{a}{x}\right)^{h} \cdot F_{a}$ is a purely inseparable extension, we would obtain that $F_{a}^{h}(z) \mid F_{a}^{h}$ is a nontrivial separable-algebraic subextension of $F_{a}(y)^{h} \mid F_{a}^{h}$. This contradicts the fact that $F_{a}^{h}$ is relatively algebraically closed in $F_{a}(y)^{h}$.

Set $\eta_{a, 0}:=y+\frac{a}{x}$. By induction on $i \in \mathbb{N}$ choose $\eta_{a, i}$ to be a root of the polynomial

$$
Y^{p}-Y-\eta_{a, i-1} \text {. }
$$

Since $v\left(y+\frac{a}{x}\right)=-1$ we obtain $v\left(\eta_{a, i}\right)=-\frac{1}{p^{i}}$ for every $i \in \mathbb{N}$. Furthermore, $v K\left(y+\frac{a}{x}\right)^{h}=v K\left(y+\frac{a}{x}\right)=\mathbb{Z}$, hence the extension $K\left(y+\frac{a}{x}\right)^{h}\left(\eta_{a, i}\right) \mid K\left(y+\frac{a}{x}\right)^{h}$ has ramification index at lest $p^{i}$. On the other hand, the degree of this extension is at most $p^{i}$. Thus the fundamental inequality shows that it has degree and ramification index $p^{i}$. Therefore,

$$
\left[K\left(y+\frac{a}{x}, \eta_{a, i}\right): K\left(y+\frac{a}{x}\right)\right]=\left[K\left(y+\frac{a}{x}\right)^{h}\left(\eta_{a, i}\right): K\left(y+\frac{a}{x}\right)^{h}\right]
$$

and the chain of the extensions $K\left(y+\frac{a}{x}, \eta_{a, i}\right)$ is linearly disjoint from $K\left(y+\frac{a}{x}\right)^{h}$ over $K\left(y+\frac{a}{x}\right)$. Moreover, $K\left(y+\frac{a}{x}\right)^{h}\left(\eta_{a, i} \mid i \in \mathbb{N}\right)$ is a separable-algebraic extension of $K\left(y+\frac{a}{x}\right)^{h}$. Since $K\left(y+\frac{a}{x}\right)^{h}$ is relatively algebraically closed in $K(x, y)^{h}$, from Lemma 2.4 we deduce that $K\left(y+\frac{a}{x}\right)^{h}\left(\eta_{a, i} \mid i \in \mathbb{N}\right)$ and $K(x, y)^{h}$ are linearly disjoint over $K\left(y+\frac{a}{x}\right)^{h}$. Hence, by Lemma 2.1 the extensions $K\left(y+\frac{a}{x}\right)\left(\eta_{a, i} \mid i \in \mathbb{N}\right)$ and $K(x, y)^{h}$ are linearly disjoint over $K\left(y+\frac{a}{x}\right)$. Using again Lemma 2.1 we deduce finally that $K(x, y)\left(\eta_{a, i} \mid i \in \mathbb{N}\right)$ is linearly disjoint from $K(x, y)^{h}$ over $K(x, y)$. Since $y$ is transcendental over $K\left(y+\frac{a}{x}\right)$ and $K\left(y+\frac{a}{x}, y\right)=K(x, y)$, the extensions $K\left(x, y, \eta_{a, j}\right) \mid K\left(x, y, \eta_{a, j-1}\right)$ remain nontrivial. We therefore obtain an infinite tower 
of Arin-Schreier extensions $K\left(x, y, \eta_{a, j}\right) \mid K\left(x, y, \eta_{a, j-1}\right)$ such that for every $j$ the valuation $v$ of $K\left(x, y, \eta_{a, j-1}\right)$ has unique extension to $K\left(x, y, \eta_{a, j}\right)$.

Since

$$
\eta_{a, 0}=a x^{-1}+\sum_{i=1}^{\infty} x^{-p^{-e_{i}}}
$$

from Lemma 4.2 by induction on $i$, if follows that each of the Artin-Schreier generators $\eta_{a, j}$ can be chosen to be of the form

$$
\eta_{a, j}=\sum_{i=j}^{\infty} c_{a, j}^{(i)} x^{-p^{-i}}
$$

with $c_{a, j}^{(i)} \in K$. Therefore $\left(K\left(x, y, \eta_{a, j}\right) \mid K(x, y), v\right)$ is a subextension of the immediate extension $\left(K\left(\left(x^{\frac{1}{p^{\infty}} \mathbb{Z}}\right)\right) \mid K(x, y), v_{x}\right)$, hence it is also immediate. It follows that $K\left(x, y, \eta_{a, j}\right) \mid K\left(x, y, \eta_{a, j-1}\right)$ is an Artin-Schreier defect extension for every $j \in \mathbb{N}$.

Setting $L_{a}:=K\left(y+\frac{a}{x}\right)\left(\eta_{a, i} \mid i \in \mathbb{N}\right)$, by Lemma 2.7 we obtain that for every two distinct elements $a, b \in E$ the infinite towers of Artin-Schreier defect extensions $K(x, y)\left(\eta_{a, i} \mid i \in \mathbb{N}\right) \mid K(x, y)$ and $K(x, y)\left(\eta_{b, i} \mid i \in \mathbb{N}\right) \mid K(x, y)$ are linearly disjoint.

Lemma 4.3. Assume that $n_{i}=-1$ for every $i \in \mathbb{N}$ in (2). For every power series

$$
\eta=\sum_{i=1}^{\infty} a_{i} x^{-p^{-i}} \in K\left(\left(x^{\frac{1}{p \infty} \mathbb{Z}}\right)\right)
$$

there is a sequence $\left(\zeta_{n}\right)_{n \in \mathbb{N}}$ of elements of $K(x, y)$ such that $v\left(\eta-\zeta_{n}\right) \geq-\frac{1}{p^{n+1}}$ for every natural number $n$.

Proof. Assume that $\eta$ is of the form 6 . We construct a sequence $\left(\zeta_{n}\right)_{n \in \mathbb{N}}$ of elements of $K(x, y)$ such that

$$
\eta-\zeta_{n}=\sum_{i=n+1}^{\infty} b_{i}^{(n)} x^{-p^{-i}}
$$

where $b_{i}^{(n)} \in K$ for $i \geq n+1$. Then, in particular, $v\left(\eta-\zeta_{n}\right) \geq-\frac{1}{p^{n+1}}$ for every natural number $n$. Set

$$
\zeta_{1}:=a_{1} y^{p^{e_{1}}-1}=a_{1} \sum_{i=1}^{\infty} x^{-p^{-e_{i}+e_{1}-1}}=a_{1} x^{-p^{-1}}+\sum_{i=2}^{\infty} a_{1} x^{-p^{-e_{i}+e_{1}-1}} .
$$

By the assumption on $\left(e_{i}\right)_{i \in \mathbb{N}}$ we have $-e_{2}+e_{1}-1 \leq-2$. Hence,

$$
\eta-\zeta_{1}=\sum_{i=2}^{\infty} a_{i} x^{-p^{-i}}-\sum_{i=2}^{\infty} a_{1} x^{-p^{-e_{i}+e_{1}-1}}=\sum_{i=2}^{\infty} b_{i}^{(1)} x^{-p^{-i}}
$$

for some $b_{i}^{(1)} \in K$. Assume now that $\zeta_{n}$ is an element of $K(x, y)$ such that equality (7) holds for some $b_{i}^{(n)} \in K$. Take $j_{n+1}:=e_{n+2}-e_{n+1}-(n+1)$. It is a nonnegative integer, since $e_{n+2}-e_{n+1} \geq(n+1)$. Putting

$$
\tilde{\zeta}_{n+1}:=\left(y^{p^{e_{n+1}}}-\sum_{i=1}^{n+1} x^{-p^{e_{n+1}-e_{i}}}\right)^{p^{j_{n+1}}} \in K(x, y)
$$


we obtain that

$$
\tilde{\zeta}_{n+1}=\left(\sum_{i=n+2}^{\infty} x^{-p^{e_{n}+1}-e_{i}}\right)^{p^{j_{n+1}}}=x^{-p^{-(n+1)}}+\sum_{i=n+3}^{\infty} x^{-p^{e_{n}+1}-e_{i}+j_{n+1}} .
$$

Set $\zeta_{n+1}:=\zeta_{n}+b_{n+1}^{(n)} \tilde{\zeta}_{n+1}$. Then

$$
\begin{aligned}
\eta-\zeta_{n+1} & =\sum_{i=n+1}^{\infty} b_{i}^{(n)} x^{-p^{-i}}-b_{n+1}^{(n)} x^{-p^{-(n+1)}}-\sum_{i=n+3}^{\infty} b_{n+1}^{(n)} x^{-p^{e_{n}+1}-e_{i}+j_{n+1}} \\
& =\sum_{i=n+2}^{\infty} b_{i}^{(n)} x^{-p^{-i}}-\sum_{i=n+3}^{\infty} b_{n+1}^{(n)} x^{-p^{e_{n+1}-e_{i}+j_{n+1}}}
\end{aligned}
$$

Since $e_{n+1}-e_{n+2}+j_{n+1}=-(n+1)$ and the sequence $\left(e_{i}\right)_{i \in \mathbb{N}}$ is strictly increasing, $e_{n+1}-e_{i}+j_{n+1} \leq-(n+2)$ for $i \geq n+3$. Therefore,

$$
\eta-\zeta_{n+1}=\sum_{i=n+2}^{\infty} b_{i}^{(n+1)} x^{-p^{-i}}
$$

where $b_{i}^{(n+1)} \in K$ for $i \geq n+2$.

By this simple observation one can easily show that each of the towers of ArtinSchreier defect extensions constructed in the proof of Theorem 1.1 consist of independent extensions.

Proof of Theorem 1.2: Take the rational function field $(K(x, y), v)$, the subfield $E$ of $K$ and elements $\eta_{a, j} \in \widehat{K(x, y)}$ as in the proof of Theorem 1.1. Since for every $a \in E$ and $i \in \mathbb{N}$ the Artin-Schreier extension $K\left(x, y, \eta_{a, j}\right) \mid K\left(x, y, \eta_{a, j-1}\right)$ has nontrivial defect, from Corollary 2.18 it follows that dist $\left(\eta_{a, j}, K\left(x, y, \eta_{a, j-1}\right)\right) \leq 0^{-}$.

On the other hand, since $\eta_{a, j}$ can be chosen to be of the form (5), from the above lemma we deduce that the set $\left(\frac{1}{p^{\infty}} \mathbb{Z}\right)^{<0}$ of all negative elements of $v K(x, y)$ is contained in the initial segment $v\left(\eta_{a, j}-K\left(x, y, \eta_{a, j-1}\right)\right)$. Consequently,

$$
\operatorname{dist}\left(\eta_{a, j}, K\left(x, y, \eta_{a, j-1}\right)\right)=0^{-} .
$$

By Corollary 2.20, this implies that $K\left(x, y, \eta_{a, j}\right) \mid K\left(x, y, \eta_{a, j-1}\right)$ is an independent Artin-Schreier defect extension.

For the construction of towers of dependent extensions we use the idea of the transformation of purely inseparable polynomials into Artin-Schreier polynomials (cf. Theorem 1.4)

Lemma 4.4. Assume that the field $K$ is perfect and the sequence $\left(n_{i} p^{-e_{i}}\right)_{i \in \mathbb{N}}$ of exponents of $y$ is bounded from above. Then for every $a \in K$ and every nonnegative integer $r$ the element $\left(\frac{y}{x^{r}}+\frac{a}{x}\right)^{1 / p}$ does not lie in the completion of $(K(x, y), v)$.

Proof. Set

$$
\gamma:=\sup \left\{n_{i} p^{-e_{i}} \mid i \in \mathbb{N}\right\}
$$

Take $a \in K$ and a nonnegative integer $r$. We show first that

$$
v\left(\left(\frac{y}{x^{r}}+\frac{a}{x}\right)^{1 / p}-f\right)<\frac{1}{p} \gamma
$$


for every $f \in K\left[x, \frac{1}{x}, y\right]$. Every such $f$ can be written in a form

$$
f=\sum_{\substack{-n \leq i \leq m \\ 0 \leq j \leq l}} a_{i j} x^{i} y^{j}
$$

for some $a_{i j} \in K$ and $m, n, l \in \mathbb{N}_{0}$. For every $i \in\{-n, \ldots, m\}$ and $j \in\{0, \ldots, l\}$, set

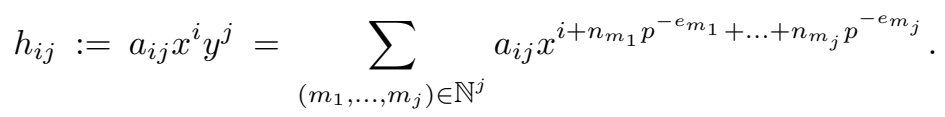

Take any $j \in\{0, \ldots, l\}$. We show that there is $N_{j} \in \mathbb{N}$ such that for every $i \in\{-n \ldots, m\}$ and $N \geq N_{j}$ we have

$$
i+n_{m_{1}} p^{-e_{m_{1}}}+\ldots+n_{m_{j}} p^{-e_{m_{j}}} \neq n_{N} p^{-e_{N}-1}-p^{-1} r
$$

for every $\left(m_{1}, \ldots, m_{j}\right) \in \mathbb{N}^{j}$ and consequently $n_{N} p^{-e_{N}-1}-p^{-1} r \notin \operatorname{supp} h_{i j}$. Since

$$
\left(\frac{y}{x^{r}}+\frac{a}{x}\right)^{1 / p}=a^{p^{-1}} x^{-p^{-1}}+\sum_{i=1}^{\infty} x^{n_{i} p^{-e_{i}-1}-p^{-1} r}
$$

the condition means that $\operatorname{supp}\left(\frac{y}{x^{r}}+\frac{a}{x}\right)^{1 / p}$ and supp $h_{i j}$ have at most finitely many common elements.

If $j=0$ then we can choose $N_{j}=1$. Let $0<j \leq l$ and suppose that

$$
i+n_{m_{1}} p^{-e_{m_{1}}}+\ldots+n_{m_{j}} p^{-e_{m_{j}}}=n_{N} p^{-e_{N}-1}-r p^{-1}
$$

for some natural numbers $m_{1}, \ldots, m_{j}$ and $N$. Without loss of generality we may assume that $m_{1} \leq \ldots \leq m_{j}$. Then, comparing denominators of both sides of the above inequality, we obtain that $e_{m_{j}} \geq e_{N}+1$ and consequently, $e_{m_{j}} \geq e_{N+1}$.

Set $d:=\min \left\{i \mid 1 \leq i<j\right.$ and $\left.m_{i}=m_{j}\right\}$ and $k:=j-d+1$. Multiplying both sides of equality (8) by $p^{e_{m_{j}}}$ we thus obtain

$$
k n_{m_{j}}=n_{N} p^{e_{m_{j}}-e_{N}-1}-r p^{e_{m_{j}}-1}-i p^{e_{m_{j}}}-\sum_{t=1}^{d-1} n_{m_{t}} p^{e_{m_{j}}-e_{m_{t}}} .
$$

Moreover, since $e_{N+1}-e_{N} \geq N$, we have $e_{m_{j}}-e_{N}-1 \geq N-1$ and

$$
e_{m_{j}}-e_{m_{s}} \geq e_{m_{j}}-e_{m_{j}-1} \geq e_{N+1}-e_{N} \geq N
$$

for every $1 \leq s<d$. By assumption, $n_{m_{j}}$ is coprime with $p$. Therefore, $p^{N-1}$ divides $k$. Choose $N_{j}$ such that $j<p^{N_{j}-1}$ and take $N \geq N_{j}$. Then, since $k \leq j$, we have that $k$ is not divisible by $p^{N-1}$ and consequently, equality (8) does not hold for any $m_{1}, \ldots, m_{j} \in \mathbb{N}$.

Hence, setting $N_{f}$ to be the maximum of the $N_{j}$ for $0 \leq j \leq l$, we have that $n_{N} p^{-e_{N}-1}-r p^{-1}$ is not an element of supp $f$ for any $N \geq N_{f}$. Therefore,

$$
v\left(\left(\frac{y}{x^{r}}+\frac{a}{x}\right)^{\frac{1}{p}}-f\right) \leq n_{N_{f}} p^{-e_{N_{f}}-1}-r p^{-1}<\frac{1}{p} \gamma .
$$

By Lemma 4.1, for every element $u$ of $K(x, y)$ there is $f \in K\left[x, \frac{1}{x}, y\right]$ such that $v(f-u)>\frac{1}{p} \gamma$. Then

$$
v\left(\left(\frac{y}{x^{r}}+\frac{a}{x}\right)^{\frac{1}{p}}-u\right)=\min \left\{v\left(\left(\frac{y}{x^{r}}+\frac{a}{x}\right)^{\frac{1}{p}}-f\right), v(f-u)\right\}<\frac{1}{p} \gamma .
$$

It follows that $\left(\frac{y}{x^{r}}+\frac{a}{x}\right)^{\frac{1}{p}} \notin K(x, y)^{c}$. 
Proof of Theorem 1.3: With the general assumptions $(3)$ on $(K(x, y), v)$, take $K$ to be a perfect field and suppose that the sequence $\left(n_{i} p^{-e_{i}}\right)_{i \in \mathbb{N}}$ of exponents of $y$ is bounded from above.

Take any $a \in K$. Define $K_{a, 0}:=K(x, y)$ and $\vartheta_{a, 0}:=y+\frac{a}{x}$. By the above lemma, $\vartheta_{a, 0}^{1 / p} \notin K_{a, 0}^{c}$. Since the value group of $K_{a, 0}$ is $p$-divisible and the residue field $K_{a, 0} v=K$ is perfect, the polynomial $Y^{p}-\vartheta_{a, 0}$ induces an immediate extension which does not lie in the completion of $K_{a, 0}$. Thus, from Theorem 1.4 we obtain that $K_{a, 0}$ admits an infinite tower of dependent Artin-Schreier defect extensions $K_{a, n} \mid K_{a, n-1}, n \in \mathbb{N}$.

From the proof of Theorem 1.4 it follows that the tower can be constructed in the following way. By induction on $n$ we choose $\vartheta_{a, n} \in \widetilde{K_{a, 0}}$ to be a root of the polynomial

$$
f_{a, n}=Y^{p}-Y-\frac{1}{b_{a, n}^{p}} \vartheta_{a, n-1}
$$

with $b_{a, n} \in K\left(y+\frac{a}{x}\right)^{\times}$of large enough value. We set $K_{a, n}:=K_{a, n-1}\left(\vartheta_{a, n}\right)$. Then, for every natural number $n$ we obtain a dependent Artin-Schreier defect extension $K_{a, n} \mid K_{a, n-1}$.

We thus have an immediate algebraic extension $F_{a}:=\bigcup_{n \in \mathbb{N}} K_{a, n}$ of $K(x, y)$, which is an infinite tower of dependent Artin-Schreier defect extensions. By the choice of $b_{a, n}$, the field $L_{a}=K\left(y+\frac{a}{x}\right)\left(\vartheta_{a, n} \mid n \in \mathbb{N}\right)$ is an algebraic extension of $K\left(y+\frac{a}{x}\right)$. From Lemma 2.7 we deduce that for two distinct elements $a, b \in K$ the extensions $F_{a} \mid K(x, y)$ and $F_{b} \mid K(x, y)$ are linearly disjoint. Hence $(K(x, y), v)$ admits $|K|$ many pairwise linearly disjoint infinite towers of dependent Artin-Schreier defect extensions. This proves the theorem in the case of an infinite field $K$.

If $K$ is finite, then repeating the above construction for $\vartheta_{r, 0}=\frac{y}{x^{r}}$ with $r \in \mathbb{N} \cup\{0\}$, we obtain an immediate extension

$$
F_{r}:=K(x, y)\left(\vartheta_{r, i} \mid i \in \mathbb{N}\right)
$$

of $K(x, y)$ being an infinite tower of dependent Artin-Schreier defect extensions $K\left(x, y, \vartheta_{r, 1}, \ldots, \vartheta_{r, i}\right) \mid K\left(x, y, \vartheta_{r, 1}, \ldots, \vartheta_{r, i-1}\right)$, where $\vartheta_{r, i}$ is a root of the polynomial

$$
Y^{p}-Y-\frac{1}{b_{r, i}^{p}} \vartheta_{r, i-1}
$$

with $b_{r, i} \in K\left(\frac{y}{x^{r}}\right)^{\times}$. From Lemma 2.8 it follows that for any two distinct $r, s \in \mathbb{N} \cup\{0\}$ the extensions $F_{r} \mid K(x, y)$ and $F_{s} \mid K(x, y)$ are linearly disjoint. Hence $(K(x, y), v)$ admits $\aleph_{0}$ many pairwise linearly disjoint infinite towers of dependent Artin-Schreier defect extensions.

Putting, as in the proofs of the previous theorems, $n_{i}=-1$ for $i \in \mathbb{N}$ we obtain the series

$$
y=\sum_{i=1}^{\infty} x^{-p^{-e_{i}}}
$$

with the sequence of exponents bounded from above by 0 . Theorems 1.2 and 1.3 imply that if $K$ is perfect, then the field $(K(x, y), v)$ admits infinite towers of both types of Artin-Schreier defect extensions. More precisely, from the theorems we obtain: 
Corollary 4.5. Assume that $K$ is a perfect field and

$$
y=\sum_{i=1}^{\infty} x^{-p^{-e_{i}}}
$$

Then the valued field $(K(x, y), v)$ admits $|K|$ many pairwise linearly disjoint infinite towers of independent and $\max \left\{|K|, \aleph_{0}\right\}$ many pairwise linearly disjoint infinite towers of dependent Artin-Schreier defect extensions.

\subsection{Valued rational function fields admitting no dependent Artin-Schreier defect extensions.}

As in the previous section we take $(K(x, y), v)$ to be a field satisfying the assumptions (3). In the foregoing constructions of Artin-Schreier defect extensions we chose $y$ to be a series with a bounded sequence of exponents $n_{i} p^{-e_{i}}$. We show that in the case of a perfect field $K$ this assumption is necessary for the existence of dependent Artin-Schreier defect extensions. Throughout this section, we assume that the sequence $\left(n_{i} p^{-e_{i}}\right)_{i \in \mathbb{N}}$ is unbounded.

Under this additional condition we obtain:

Lemma 4.6. For every natural number $n$ the elements $x^{p^{-n}}$ and $y^{1 / p}$ lie in the completion of $K(x, y)$.

Proof. We show first that $x^{p^{-N}} \in K(x, y)^{c}$ for every natural number $N$. Take $N, j \in \mathbb{N}$ with $j \geq N$. Set $s_{j}:=e_{j+1}-e_{j}-N$. By assumption, $e_{j+1}-e_{j} \geq j$, thus $s_{j}$ is a nonnegative integer. Set

$$
\begin{aligned}
\widetilde{\xi_{j}} & :=\left(y^{p^{e_{j}}}-\sum_{i=1}^{j} x^{n_{i} p^{e_{j}-e_{i}}}\right)^{p^{s_{j}}}=\left(\sum_{i=j+1}^{\infty} x^{n_{i} p^{e_{j}-e_{i}}}\right)^{p^{s_{j}}} \\
& =x^{n_{j+1} p^{-N}}+\sum_{i=j+2}^{\infty} x^{n_{i} p^{s_{j}+e_{j}-e_{i}}} .
\end{aligned}
$$

Since $p$ and $n_{j+1}$ are coprime, there are integers $l$ and $k>0$ such that

$$
k n_{j+1}+l p^{N}=1 \text {. }
$$

Hence, putting $\xi_{j}:=x^{l}\left(\widetilde{\xi}_{j}\right)^{k}$ we obtain

$$
\xi_{j}=x^{l}\left(x^{n_{j+1} p^{-N}}+\sum_{i=j+2}^{\infty} x^{n_{i} p^{s_{j}+e_{j}-e_{i}}}\right)^{k}=x^{l+k n_{j+1} p^{-N}}+\ldots=x^{p^{-N}}+\ldots,
$$

where

$$
\begin{aligned}
v\left(\xi_{j}-x^{p^{-N}}\right) & =l+(k-1) n_{j+1} p^{-N}+n_{j+2} p^{e_{j}+s_{j}-e_{j+2}} \\
& =p^{-N}-n_{j+1} p^{-N}+n_{j+2} p^{-N-e_{j+2}+e_{j+1}} \\
& =p^{-N}+p^{-N+e_{j+1}}\left(-n_{j+1} p^{-e_{j+1}}+n_{j+2} p^{-e_{j+2}}\right) .
\end{aligned}
$$

Note that $-n_{j+1} p^{-e_{j+1}}+n_{j+2} p^{-e_{j+2}}>p^{-j}$ for infinitely many $j \geq N$. Indeed, suppose $-n_{j+1} p^{-e_{j+1}}+n_{j+2} p^{-e_{j+2}} \leq p^{-j}$ for all but finitely many $j$. Then the fact that $-n_{j+1} p^{-e_{j+1}}+n_{j+2} p^{-e_{j+2}}>0$ and the series $\sum_{j=1}^{\infty} p^{-j}$ is convergent contradicts 
the assumption that the sequence $\left(n_{j} p^{-e_{j}}\right)_{j \in \mathbb{N}}$ is unbounded. Therefore,

$$
v\left(\xi_{j}-x^{p^{-N}}\right) \geq p^{-N}+p^{-N} p^{e_{j+1}-j} \geq p^{-N}+p^{-N} p^{e_{j}}
$$

for infinitely many $j \geq N$. By assumption $\left(e_{j}\right)_{j \in \mathbb{N}}$ is a strictly increasing sequence of natural numbers, hence for arbitrary large elements $\gamma \in \frac{1}{p^{\infty}} \mathbb{Z}$ we can choose $j \geq N$ such that $v\left(x^{p^{-N}}-\xi_{j}\right)>\gamma$. Thus $x^{p^{-N}} \in K(x, y)^{c}$.

Consider now the element $y^{1 / p}$. Since $\left(n_{j} p^{-e_{j}}\right)_{j \in \mathbb{N}}$ is a strictly increasing unbounded sequence and

$$
v\left(y^{1 / p}-\sum_{i=1}^{k} x^{n_{i} p^{-e_{i}-1}}\right)=n_{k+1} p^{-e_{k+1}-1}=p^{-1}\left(n_{k+1} p^{-e_{k+1}}\right)
$$

for every $k \in \mathbb{N}$, the values are cofinal in $\frac{1}{p^{\infty}} \mathbb{Z}$. By what we have shown,

$$
\sum_{i=1}^{k} x^{n_{i} p^{-e_{i}-1}} \in K(x, y)^{c}
$$

Therefore also $y^{1 / p}$ lies in the completion of $K(x, y)$.

Proposition 4.7. If the field $K$ is perfect then $(K(x, y), v)$ admits no dependent Artin-Schreier defect extensions.

Proof. Since $K$ is perfect, by the above lemma $K(x, y)^{1 / p} \subseteq K(x, y)^{c}$. Then, from Theorem 1.4 if follows that $K(x, y)$ admits no dependent Artin-Schreier defect extensions.

Nevertheless, the next proposition shows that the field $(K(x, y), v)$ can still admit independent Artin-Schreier defect extensions.

Proposition 4.8. Assume that the element $y$ is of positive value. If $K$ admits a perfect subfield of cardinality $\kappa$ then $(K(x, y), v)$ admits $\kappa$ many pairwise linearly disjoint infinite towers of independent Artin-Schreier defect extensions.

Proof. Take an extension of the valuation $v$ to the algebraic closure of $K(x, y)$ and denote it again by $v$. Since $y$ is a pseudo limit of a pseudo Cauchy sequence of transcendental type in $L:=K\left(x^{p^{-i}} \mid i \in \mathbb{N}\right)$, then by Lemma 2.11 the field $L^{h}$ is relatively algebraically closed in $L(y)^{h}$. Furthermore, $L^{h}=K(x)^{h}$. $L$ is a purely inseparable extension of $K(x)$ and $K(x, y)^{h} \mid K(x)^{h}$ is separable. Hence, using the fact that $K(x, y)^{h} \subseteq L(y)^{h}$ we deduce that $K(x)^{h}$ is relatively algebraically closed in $K(x, y)^{h}$. Indeed, if there were an element $z \in K(x, y)^{h} \backslash K(x)^{h}$ algebraic over $K(x)^{h}$, then $z$ would be separable over $K(x)^{h}$. Thus $L^{h}(z) \mid L^{h}$ would be a nontrivial separable-algebraic subextension of $L(y)^{h} \mid L^{h}$, a contradiction.

Assume that $E$ is a perfect subfield of cardinality $\kappa$ and take $a \in E$. Define $K_{a, 0}:=K(x, y), \xi_{a, 0}:=\frac{a}{x}$ and by induction on $n$ choose $\xi_{a, n}$ to be a root of the polynomial

$$
Y^{p}-Y-\xi_{a, n-1} .
$$

Set $K_{a, n}:=K_{a, n-1}\left(\xi_{a, n}\right)=K\left(x, y, \xi_{a, n}\right)$. Since $v\left(\frac{a}{x}\right)=-1$, for every natural number $n$ we obtain $v\left(\xi_{a, n}\right)=-\frac{1}{p^{n}}$. Therefore, from the fact that $v K(x)^{h}=\mathbb{Z}$ we obtain

$$
\left(v K(x)^{h}\left(\xi_{a, n}\right): v K(x)^{h}\right) \geq p^{i} .
$$


On the other hand, the degree of the extension $K(x)^{h}\left(\xi_{a, n}\right) \mid K(x)^{h}$ is at most $p^{i}$. Hence the fundamental inequality shows that it has degree and ramification index $p^{i}$. Consequently, chain of the extensions $K\left(x, \xi_{a, i}\right)$ is linearly disjoint from $K(x)^{h}$ over $K(x)$. Furthermore, $K(x)^{h}\left(\xi_{a, i} \mid i \in \mathbb{N}\right)$ is a separable-algebraic extension of $K(x)^{h}$. As we have shown, $K(x)^{h}$ is relatively algebraically closed in $K(x, y)^{h}$. Thus from Lemma 2.4 we deduce that $K(x)^{h}\left(\xi_{a, i} \mid i \in \mathbb{N}\right)$ and $K(x, y)^{h}$ are linearly disjoint over $K(x)^{h}$. Hence, by Lemma 2.1 the extensions $K(x)\left(\xi_{a, i} \mid i \in \mathbb{N}\right) \mid K(x)$ and $K(x, y)^{h} \mid K(x)$ are linearly disjoint. Using again Lemma 2.1 we deduce finally that $K(x, y)\left(\xi_{a, i} \mid i \in \mathbb{N}\right)$ is linearly disjoint from $K(x, y)^{h}$ over $K(x, y)$. Since $y$ is transcendental over $K(x)$ and $\left[K\left(x, \xi_{a, n}\right): K(x)\right]=p^{n}$, we obtain that also each of the extensions $K_{a, n} \mid K(x, y)$ has degree $p^{n}$ and, as we have shown, is linearly disjoint from $K(x, y)^{h} \mid K(x, y)$.

As $\xi_{a, 0}=a x^{-1}$, from Lemma 4.2 by induction on $n$ it follows that each of the Artin-Schreier generators $\xi_{a, n}$ can be chosen to be of the form

$$
\xi_{a, n}=\sum_{i=n}^{\infty} d_{a, n}^{(i)} x^{-p^{-i}} .
$$

Hence $K(x, y) \subseteq K_{a, n} \subseteq K\left(\left(x^{\frac{1}{p^{\infty}} \mathbb{Z}}\right)\right)$. The fact that $\left(K\left(\left(x^{\frac{1}{p^{\infty}} \mathbb{Z}}\right)\right) \mid K(x, y), v_{x}\right)$ is an immediate extension implies that also $\left(K_{a, n} \mid K(x, y), v\right)$ is immediate.

Set now $\eta_{a, 0}:=y+\frac{a}{x}$. Since $v \eta_{a, 0}=-1$ we deduce that $v K\left(\eta_{a, 0}\right)=\mathbb{Z}$. By induction on $n$ we choose $\eta_{a, n}$ to be a root of the polynomial

$$
Y^{p}-Y-\eta_{a, n-1}
$$

Then we obtain $v \eta_{a, n}=-\frac{1}{p^{n}}$ and as in the proof of Theorem 1.1 we deduce that $\left(K\left(y+\frac{a}{x}, \eta_{a, n}\right) \mid K\left(y+\frac{a}{x}, \eta_{a, n-1}\right), v\right)$ is an Artin-Schreier extension of ramification index $p$. Since $y$ is transcendental over $K\left(y+\frac{a}{x}\right)$ and $K\left(y+\frac{a}{x}, y\right)=K(x, y)$, the extension $K\left(x, y, \eta_{a, n}\right) \mid K\left(x, y, \eta_{a, n-1}\right)$ remain nontrivial.

We use now the properties of the constructed extensions $K_{a, n} \mid K_{a, n-1}$ to show that $K\left(x, y, \eta_{a, n}\right) \mid K\left(x, y, \eta_{a, n-1}\right)$ form an infinite tower of independent Artin-Schreier defect extensions. By the additivity of Artin-Schreier polynomials, for every $n \in \mathbb{N}$ we can choose $\eta_{a, n}$ to be of the form

$$
\eta_{a, n}=\xi_{n}+\xi_{a, n},
$$

where $\xi_{n}$ is a root of the polynomial $Y^{p}-Y-\xi_{n-1}$ with $\xi_{0}=y$. Since $v \xi_{0}>0$ from Lemma 2.16 if follows that we can choose the Artin-Schreier generators $\xi_{n}$ in such a way that $v \xi_{n}>0$ for every $n \in \mathbb{N}$. Consider the henselizations of $K\left(x, y, \xi_{n}\right)$ with respect to the fixed extension of the valuation $v$ of $K(x, y)$ to $\widehat{K(x, y)}$. Then $K\left(x, y, \xi_{n}\right)^{h}=K(x, y)^{h}$ for every natural number $n$. Indeed, since $v \xi_{0}>0$, by Lemma 2.17 the Artin-Schreier generator $\xi_{1}$ lies in the henselization of $K(x, y)$. Thus $K\left(x, y, \xi_{1}\right)^{h}=K(x, y)^{h}$. Take any $n \in \mathbb{N}$ and assume that $K\left(x, y, \xi_{n}\right)^{h}=K(x, y)^{h}$. By our choice, $v \xi_{n}>0$ hence using again Lemma 2.17 we deduce that

$$
K\left(x, y, \xi_{n+1}\right)^{h}=K\left(x, y, \xi_{n}\right)^{h}\left(\xi_{n+1}\right)=K\left(x, y, \xi_{n}\right)^{h}=K(x, y)^{h} .
$$

Set $L_{a, 0}:=K(x, y)$ and $L_{a, n}:=L_{a, n-1}\left(\eta_{a, n}\right)$ for every $n \in \mathbb{N}$. We show that for every natural number $n$ the extension $L_{a, n} \mid L_{a, n-1}$ is linearly disjoint from 
$L_{a, n-1}^{h} \mid L_{a, n-1}$. Since $\xi_{1} \in K(x, y)^{h}$ we obtain that

$$
L_{a, 1}^{h}=K(x, y)^{h}\left(\xi_{1}+\xi_{a, 1}\right)=K(x, y)^{h}\left(\xi_{a, 1}\right)=K_{a, 1}^{h} .
$$

Take a natural number $n$ and assume that $L_{a, n}^{h}=K_{a, n}^{h}$. As we have shown $\xi_{n+1} \in K(x, y)^{h} \subseteq L_{a, n}^{h}$, hence

$$
L_{a, n+1}^{h}=L_{a, n}^{h}\left(\xi_{n+1}+\xi_{a, n+1}\right)=L_{a, n}^{h}\left(\xi_{a, n+1}\right)=K_{a, n}^{h}\left(\xi_{a, n+1}\right)=K_{a, n+1}^{h} .
$$

Therefore, by induction we obtain the equality $L_{a, n}^{h}=K_{a, n}^{h}$ for every $n \in \mathbb{N}$.

Suppose the Artin-Schreier extension $L_{a, n} \mid L_{a, n-1}$ was not linearly disjoint from $L_{a, n-1}^{h} \mid L_{a, n-1}$ for some $n \in \mathbb{N}$. Then $\eta_{a, n} \in L_{a, n-1}^{h}$. Since $\eta_{a, n}=\xi_{n}+\xi_{a, n}$ and $\xi_{n} \in K(x, y)^{h} \subseteq L_{a, n-1}^{h}$, we would have that $\xi_{a, n} \in L_{a, n-1}^{h}=K_{a, n-1}^{h}$. On the other hand, we have proved that the valuation $v$ of $K_{a, n-1}$ has a unique extension to the field $K_{a, n}=K_{a, n-1}\left(\xi_{a, n}\right)$, a contradiction. Therefore the valuation $v$ of $L_{a, n-1}$ has a unique extension to the field $L_{a, n}$ for every $n \in \mathbb{N}$.

Since the value group of $K(x, y)$ is $p$-divisible, each of the extensions $L_{a, n} \mid L_{a, n-1}$ has ramification index equal to 1 . Take a natural number $n$. Using the fact that the henselization is an immediate field extension, we obtain that

$$
L_{a, n} v=L_{a, n}^{h} v=K_{a, n}^{h} v=K_{a, n} v=K .
$$

Therefore the extension $L_{a, n} \mid L_{a, n-1}$ is immediate. Consequently, each of the ArtinSchreier extensions $L_{a, n} \mid L_{a, n-1}$ has nontrivial defect.

Take a natural number $n$. From Lemma 2.18 it follows that dist $\left(\eta_{a, n}, L_{a, n-1}\right) \leq 0^{-}$. Note that

$$
\eta_{a, n}=\sum_{i=n}^{\infty} d_{a, n}^{(i)} x^{-p^{-i}}+\xi_{n}
$$

with $v \xi_{n}>0$. By Lemma 4.6, for every $j \geq n$ we have that

$$
\sum_{i=n}^{j} d_{a, n}^{(i)} x^{-p^{-i}} \in K(x, y)^{c} .
$$

Thus there is $u_{n, j} \in K(x, y)$ such that $v\left(\sum_{i=n}^{j} d_{a, n}^{(i)} x^{-p^{-i}}-u_{n, j}\right)>0$. Then

$v\left(\eta_{a, n}-u_{n, j}\right)=v\left(\left(\sum_{i=n}^{j} d_{a, n}^{(i)} x^{-p^{-i}}-u_{n, j}+\xi_{n}\right)+\sum_{i=j+1}^{\infty} d_{a, n}^{(i)} x^{-p^{-i}}\right) \geq-p^{-(j+1)}$.

Therefore the set of values $v\left(\eta_{a, n}-u_{n, j}\right)$ is cofinal in $\left(\frac{1}{p^{\infty}} \mathbb{Z}\right)^{<0}$. Consequently $\operatorname{dist}\left(\eta_{a, n}, L_{a, n-1}\right)=0^{-}$. From Corollary 2.20 it follows that $L_{a, n} \mid L_{a, n-1}$ is an independent Artin-Schreier defect extension.

Using Lemma 2.7 we obtain that for any two distinct elements $a, b \in E$ the extensions $\bigcup_{n \in \mathbb{N}} L_{a, n} \mid K(x, y)$ and $\bigcup_{n \in \mathbb{N}} L_{b, n} \mid K(x, y)$ are linearly disjoint.

Assume that the field $K$ is perfect and $v y>0$. Then from Proposition 4.7 it follows that $(K(x, y), v)$ admits no dependent Artin-Schreier defect extensions. However, by Proposition 4.8 the field admits $|K|$ many pairwise linearly disjoint infinite towers of independent Artin-Schreier defect extensions.

Note also that the above construction of towers $\bigcup_{n \in \mathbb{N}} L_{a, n} \mid K(x, y)$ of ArtinSchreier defect extensions does not depend on the fact that the sequence $\left(n_{i} p^{-e_{i}}\right)_{i \in \mathbb{N}}$ 
of exponents of $y$ is unbounded. We use the assumption only to show that all of the Artin-Schreier defect extensions in the towers are independent. If the sequence of exponents of $y$ is bounded from above, we are not yet able to classify the constructed Artin-Schreier extensions. Nevertheless, we obtain the following corollary to the proof of Proposition 4.8:

Corollary 4.9. Take a valued rational function field $(K(x, y), v)$ satisfying conditions (3). We assume that the element $y$ is of positive value, but not necessarily that its exponents are unbounded. If $K$ admits a perfect subfield of cardinality $\kappa$, then $(K(x, y), v)$ admits $\kappa$ many pairwise linearly disjoint infinite towers of ArtinSchreier defect extensions.

Therefore, the construction of towers of Artin-Schreier defect extensions as in the proof of Proposition 4.8 gives us another proof of Theorem 1.1.

\section{5. $p$-ELEMENTARY EXTENSIONS OF RATIONAL FUNCTION FIELDS}

Take a field $L$ of characteristic $p>0$. A polynomial $f \in L[X]$ is called a p-polynomial if $f=\mathcal{A}+c$, where $\mathcal{A}$ is an additive polynomial and $c$ is a constant. An important example of $p$-polynomials are Artin-Schreier polynomials $X^{p}-X-c$ with $c \in L$. If an Artin-Schreier polynomial is irreducible, each of its roots generates a Galois extension of degree $p$.

We consider now a more general class of Galois extensions of degree a power of $p$. An algebraic extension $L^{\prime} \mid L$ is called a p-elementary extension if it is a finite Galois extension and its Galois group $\operatorname{Gal}\left(L^{\prime} \mid L\right)$ is an elementary-abelian p-group, that is, $\operatorname{Gal}\left(L^{\prime} \mid L\right)$ is an abelian $p$-group such that every nonzero element of the group has order $p$; if $\left[L^{\prime}: L\right]=p^{n}$, then the group is a direct sum of $n$ cyclic subgroups of order $p$. Hence $L^{\prime} \mid L$ is a compositum of $n$ many parallel Galois extensions of degree $p$, thus a tower of Artin-Schreier extensions. Every Artin-Schreier extension is generated by a root of a $p$-polynomial of degree $p$ over $L$. More generally, one can show that every $p$-elementary extension is generated by a root of some irreducible p-polynomial (cf. Theorem 34 of [Ku2]).

Take any natural number $n$ and assume that $\mathbb{F}_{p^{n}} \subseteq L$. Consider the polynomial

$$
f=X^{p^{n}}-X-a \in L[X] .
$$

Note that for $n=1$ we obtain an Artin-Schreier polynomial. Assume that $f$ is irreducible over $L$ and consider the extension $L(\vartheta) \mid L$ generated by a root $\vartheta$ of $f$. Since the elements $\vartheta+c$ with $c \in \mathbb{F}_{p^{n}}$ form the set of all roots of $f$, the extension $L(\vartheta) \mid L$ is normal, hence Galois. Furthermore,

$$
\operatorname{Gal}(L(\vartheta) \mid L)=\left\{\sigma_{c} \mid c \in \mathbb{F}_{p^{n}}\right\},
$$

where $\sigma_{c}(\vartheta)=\vartheta+c$. Thus the Galois group of $L(\vartheta) \mid L$ is an elementary-abelian $p$-group. Consequently, $L(\vartheta) \mid L$ is a $p$-elementary extension of degree $p^{n}$. As in the case of Artin-Schreier extensions, for the extensions of valued fields generated by roots of polynomials $X^{p^{n}}-X-a$ we obtain the following facts.

Lemma 5.1. Assume that $(L, v)$ is a valued field and $\vartheta$ a root of the polynomial $f=X^{p^{n}}-X-a \in L[X]$. If $v a \leq 0$, then $v \vartheta=\frac{1}{p^{n}} v a$. If $v a>0$, then exactly one of the conjugates of $\vartheta$ has value va and the other roots of $f$ have value 0 . 
Proof. If $v \vartheta \neq 0$ then $v \vartheta^{p^{n}} \neq \vartheta$. Thus from equality $\vartheta^{p^{n}}-\vartheta=a$ it follows that $v a=\min \left\{v \vartheta, p^{n} v \vartheta\right\}$. Therefore, if $v a=0$, we have that $v \vartheta=0$. Assume that $v a<0$. Then also $v \vartheta<0$ and consequently $v a=p^{n} v \vartheta$. Thus $v \vartheta=\frac{1}{p^{n}} v a$. Note that

$$
a=\vartheta^{p^{n}}-\vartheta=\prod_{c \in \mathbb{F}_{p^{n}}}(\vartheta+c)
$$

Thus, if $v a>0$, there must be a conjugative $\vartheta^{\prime}$ of $\vartheta$ of positive value. Since $v\left(\vartheta^{\prime}+c\right)=0$ for every $c \in \mathbb{F}_{p^{n}}^{*}$, the other roots of $f$ have value 0 .

Lemma 5.2. Assume that $(L, v)$ is a valued field of positive characteristic $p$ and $\mathbb{F}_{p^{n}} \subseteq$ Lv for some $n \in \mathbb{N}$. Take a polynomial $f=X^{p^{n}}-X-a \in L[X]$. If va $>0$ or va $=0$ and $X^{p^{n}}-X-a v$ has a root in Lv then every root of $f$ lies in the henselization of $L$ (with respect to every extension of $v$ to $\tilde{L}$ ).

Proof. If $v a>0$, then the polynomial $X^{p^{n}}-X$ is the reduction of $f$ modulo $v$. Since $\mathbb{F}_{p^{n}} \subseteq L v$, the polynomial $X^{p^{n}}-X$ splits completely in $L v$. Assume that $v a=0$ and $X^{p^{n}}-X-a v$ has a root $\vartheta$ in $L v$. Since all other roots of the polynomial are of the form $\vartheta+c$ with $c \in \mathbb{F}_{p^{n}}$, also in this case the reduction of $f$ modulo $v$ splits completely in $L v$. Therefore, in both cases it follows from Hensel's Lemma that $X^{p^{n}}-X-a$ splits completely in every henselization of $(L, v)$.

The similarities between the Artin-Schreier extensions and the more general class of $p$-elementary extensions generated by roots of polynomials $X^{p^{n}}-X-a$ give rise to the question if we can use the techniques from Theorems 1.1 and 1.3 to construct towers of $p$-elementary extensions of degree and defect $p^{n}$. The next theorem shows that in the case of constructions from the proof of Theorem 1.1 such generalization is possible.

Theorem 5.3. Take a field $K$ of positive characteristic $p$ and a natural number $n$ such that $\mathbb{F}_{p^{n}} \subseteq K$. Assume that $K$ contains a perfect subfield of cardinality $\kappa$. Then there is a valuation $v$ of the rational function field $K(x, y) \mid K$, trivial on $K$, such that $(K(x, y), v)$ admits $\kappa$ many pairwise linearly disjoint infinite towers of p-elementary extensions of degree and defect $p^{n}$.

Proof. Take $(K(x, y), v)$ to be the valued rational function field defined as in the proof of Theorem 1.1. Namely, we assume that $(K(x, y), v)$ satisfies assumptions (3) with $n_{i}=-1$ for every $i \in \mathbb{N}$ in (2). Then $y$ is of the form

$$
y=\sum_{i=1}^{\infty} x^{-p^{-e_{i}}} .
$$

Take a natural number $n$ such that $\mathbb{F}_{p^{n}} \subseteq K$. Suppose that $E$ is a perfect subfield of $K$ of cardinality $\kappa$ and choose $a \in E$. From the proof of Theorem 1.1 we know that $y$ is a pseudo limit of a pseudo Cauchy sequence of transcendental type in the perfect hull $F_{a}=K\left(y+\frac{a}{x}\right)^{1 / p^{\infty}}$ of $K\left(y+\frac{a}{x}\right)$ and consequently, $K\left(y+\frac{a}{x}\right)^{h}$ is relatively algebraically closed in $K(x, y)^{h}$.

Set $\eta_{a, 0}^{(n)}:=y+\frac{a}{x}$. By induction on $i \in \mathbb{N}$ choose $\eta_{a, i}^{(n)}$ to be a root of the polynomial

$$
Y^{p^{n}}-Y-\eta_{a, i-1}^{(n)}
$$


Since $v\left(y+\frac{a}{x}\right)=-1$ we obtain that $v K\left(y+\frac{a}{x}\right)^{h}=v K\left(y+\frac{a}{x}\right)=\mathbb{Z}$ and $v \eta_{a, i}^{(n)}=-\frac{1}{p^{i n}}$ for every $i \in \mathbb{N}$. Therefore, using similar arguments as in the proof of Theorem 1.1 we deduce that

$$
\left[K\left(y+\frac{a}{x}\right)^{h}\left(\eta_{a, i}^{(n)}\right): K\left(y+\frac{a}{x}\right)^{h}\right]=p^{i n}
$$

and consequently $K\left(x, y, \eta_{a, i}^{(n)}\right) \mid K\left(x, y, \eta_{a, i-1}^{(n)}\right)$ is an extension of degree $p^{n}$ such that the valuation $v$ of $K\left(x, y, \eta_{a, i-1}^{(n)}\right)$ has a unique extension to the field $K\left(x, y, \eta_{a, i}^{(n)}\right)$ for every $i \in \mathbb{N}$. Since $\left[K\left(x, y, \eta_{a, i}^{(n)}\right): K\left(x, y, \eta_{a, i-1}^{(n)}\right)\right]=p^{n}$, the polynomial $Y^{p^{n}}-Y-\eta_{a, i-1}^{(n)}$ is irreducible. Thus, $K\left(x, y, \eta_{a, i}^{(n)}\right) \mid K\left(x, y, \eta_{a, i-1}^{(n)}\right)$ is a $p$-elementary extension for every $i \in \mathbb{N}$.

Note that the element $\eta_{a, 0}^{(n)}$ is of the form

$$
\eta_{a, 0}^{(n)}=a x^{-1}+\sum_{i=1}^{\infty} x^{-p^{-e_{i}}},
$$

thus from Lemma 4.2 by induction on $i$, it follows that each of the generators $\eta_{a, j}^{(n)}$ can be chosen to be of the form

$$
\eta_{a, j}^{(n)}=\sum_{i=n j}^{\infty} c_{a, j}^{(n)}(i) x^{-p^{-i}}
$$

with $c_{a, j}^{(n)}(i) \in K$. Therefore $\left(K\left(x, y, \eta_{a, j}^{(n)}\right) \mid K(x, y), v\right)$, as a subextension of the immediate extension $\left(K\left(\left(x^{\frac{1}{p^{\infty}} \mathbb{Z}}\right)\right) \mid K(x, y), v_{x}\right)$, is also immediate. Hence we obtain an infinite tower of $p$-elementary extensions $K\left(x, y, \eta_{a, j}^{(n)}\right) \mid K\left(x, y, \eta_{a, j-1}^{(n)}\right)$ of degree and defect $p^{n}$.

Finally, from Lemma 2.7 it follows that for every two distinct $a, b \in E$ the extensions $K(x, y)\left(\eta_{a, i}^{(n)} \mid i \in \mathbb{N}\right) \mid K(x, y)$ and $K(x, y)\left(\eta_{b, i}^{(n)} \mid i \in \mathbb{N}\right) \mid K(x, y)$ are linearly disjoint.

Note that since every $p$-elementary extension is a tower of Artin-Schreier extensions, $K(x, y)\left(\eta_{a, i}^{(n)} \mid i \in \mathbb{N}\right) \mid K(x, y)$ is in particular an infinite tower of Artin-Schreier defect extensions.

Consider now the methods used in the proof of Theorem 1.3, or more generally in the proof of Theorem 1.4, to show the existence of infinite towers of dependent Artin-Schreier defect extensions. The constructions of dependent extensions are based on the deformation of purely inseparable polynomials into Artin-Schreier polynomials. Take a valued field $(L, v)$ of characteristic $p>0$. From Theorem 2.21 we know that a suitable deformation of a purely inseparable polynomial $X^{p}-\eta^{p} \in L[X]$ with $\eta \notin L^{c}$ into a polynomial $X^{p}-X-\left(\frac{\eta}{b}\right)^{p}$ yields a dependent Artin-Schreier defect extension generated by a root $\vartheta$ of the Artin-Schreier polynomial. The fact that $(L(\vartheta) \mid L, v)$ has nontrivial defect follows from the relation $\vartheta \sim_{L} \frac{\eta}{b}$ between the generators of the purely inseparable and the Artin-Schreier extension. Indeed, if the extension of the valuation $v$ of $L$ to $L(\vartheta)$ were not unique, the Artin-Schreier generator $\vartheta$ would lie in the henselization $L^{h}$ of $L$ with respect to some extension of $v$ to $\tilde{L}$. Since $\vartheta \sim_{L} \frac{\eta}{b}$, by Theorem 2.14 we would obtain that $\frac{\eta}{b}$ cannot be purely inseparable over $L$, a contradiction. By Lemma 2.21 of 
[Ku3] we obtain that the extension $(L(\vartheta) \mid L, v)$ is immediate and consequently has nontrivial defect.

The following lemma shows that also a suitable deformation of purely inseparable polynomials of higher degrees into separable p-polynomials induces a relation between roots of the two polynomials.

Lemma 5.4. Assume that $(L, v)$ is a valued field of positive characteristic $p$. Take an immediate purely inseparable extension $L(\xi) \mid L$ of degree at most $p^{n}$ such that $\xi \notin L^{c}$. Set $\varepsilon:=\operatorname{dist}(\xi, L)$. Then for every $b \in L^{\times}$such that

$$
\left(p^{n}-1\right) v b+v \xi>p^{n} \varepsilon,
$$

a root $\vartheta$ of the polynomial

$$
g_{b}=X^{p^{n}}-X-\left(\frac{\xi}{b}\right)^{p^{n}}
$$

satisfies the condition

$$
\vartheta \sim_{L} \frac{\xi}{b}
$$

Proof. Take $b \in L^{\times}$satisfying (9) and a root $\vartheta$ of the polynomial

$$
g_{b}=X^{p^{n}}-X-\left(\frac{\xi}{b}\right)^{p^{n}} \in L[X] .
$$

Since $L(\xi) \mid L$ is immediate, by Theorem 2.13 we have that $v \xi<\varepsilon$ and therefore,

$$
\left(p^{n}-1\right) v b+v \xi>p^{n} \varepsilon>p^{n} v \xi .
$$

Thus $v b>v \xi$ and consequently $v\left(\frac{\xi}{b}\right)<0$. Hence

$$
v \vartheta=\frac{1}{p^{n}} v\left(\frac{\xi}{b}\right)^{p^{n}}=v \xi-v b .
$$

By definition of $\vartheta$ we obtain

$$
\xi^{p^{n}}+b^{p^{n}} \vartheta==\xi^{p^{n}}+b^{p^{n}}\left(\vartheta^{p^{n}}-\left(\frac{\xi}{b}\right)^{p^{n}}\right)=(b \vartheta)^{p^{n}} .
$$

Take any $c \in L$. By equality (11), assumption on $v b$ and definition of $\varepsilon$ we obtain $v\left(b^{p^{n}} \vartheta\right)=p^{n} v b+v \xi-v b=\left(p^{n}-1\right) v b+v \xi>p^{n} \varepsilon \geq p^{n} v(\xi-c)=v\left(\xi^{p^{n}}-c^{p^{n}}\right)$.

By (12) this implies that

$$
\begin{aligned}
v(\xi-c) & =\frac{1}{p^{n}} v\left(\xi^{p^{n}}-c^{p^{n}}\right)=\frac{1}{p^{n}} \min \left\{v\left(\xi^{p^{n}}-c^{p^{n}}\right), v\left(b^{p^{n}} \vartheta\right)\right\} \\
& =\frac{1}{p^{n}} v\left(\xi^{p^{n}}-c^{p^{n}}+b^{p^{n}} \vartheta\right)=\frac{1}{p^{n}} v\left((b \vartheta)^{p^{n}}-c^{p^{n}}\right)=v(b \vartheta-c) .
\end{aligned}
$$

Since $L(\xi) \mid L$ is immediate, by Theorem 2.13 the set $v(\xi-L)$ has no maximal element. Hence from Lemma 2.12 we deduce that $b \vartheta \sim_{L} \xi$ and thus,

$$
\vartheta \sim_{L} \frac{\xi}{b}
$$


Nevertheless, the next example shows that in the case of extensions of degree higher that the characteristic of the field, the relation (10) does not suffice to prove that $L(\vartheta) \mid L$ is disjoint from the henselization. Therefore, the direct generalization of Theorem 2.21 to the purely inseparable extensions of higher degrees is not possible.

Example 5.5. Take a perfect field $K$ of characteristic $p>0$ and consider the valued rational function field $(K(x, y), v)$ defined in Section 4 by the conditions $(3)$. Assume that $n_{i}=-1$ for every $i \in \mathbb{N}$ in (2). Then

$$
y=\sum_{i=1}^{\infty} x^{-p^{-e_{i}}} .
$$

Take any extension of $v$ to $\widetilde{K(x, y)}$ and denote by $K(x, y)^{h}$ the henselization of $K(x, y)$ with respect to $v$. We construct a $p$-elementary extension $K(x, y, \vartheta) \mid K(x, y)$ of degree $p^{2}$ generated by a root $\vartheta$ of some polynomial $X^{p^{2}}-X-z$ such that $K(x, y, \vartheta) \mid K(x, y)$ is not linearly disjoint from $K(x, y)^{h} \mid K(x, y)$, but can be derived from an immediate purely inseparable extension of degree $p^{2}$, which does not lie in the completion of $K(x, y)$.

Set $d:=y^{-p^{e_{1}}} \in K(y)$ and consider the Artin-Schreier polynomial

$$
h_{1}:=Y^{p}-Y-\frac{1}{d^{p}} y .
$$

Since the value group of $K(x, y)$ is $p$-divisible and the residue field $K(x, y) v=K$ is perfect, by Lemma 4.4 the polynomial $Y^{p}-y$ induces an immediate purely inseparable extension, which does not lie in the completion $K(x, y)^{c}$ of $K(x, y)$. Moreover, from the proof of Lemma 4.4 it follows that $\operatorname{dist}\left(y^{1 / p}, K(x, y)\right) \leq 0^{-}$. Since $v y=-\frac{1}{p^{e_{1}}}$ and $v d=1$ we have that

$$
(p-1) v d+v y>0 \geq p \operatorname{dist}\left(y^{1 / p}, K(x, y)\right) .
$$

Thus the element $d$ satisfies the condition (1) of Theorem 2.21 and consequently the polynomial $h_{1}$ induces a dependent Artin-Schreier defect extension $K(x, y, \eta) \mid K(x, y)$. Note that $\eta$ is a root of the polynomial

$$
\tilde{h}_{1}:=Y^{p^{2}}-Y-\frac{1}{d^{p}} y-\frac{1}{d^{p^{2}}} y^{p} .
$$

Set $n=p^{e_{1}+2}+1$ and take a $p$-polynomial

$$
h_{2}:=Y^{p^{2}}-Y-\frac{1}{d^{p^{2}}} y^{-n} .
$$

Since $v \frac{1}{d^{p^{2}}} y^{-n}=-p^{2}+\frac{n}{p^{e_{1}}}=\frac{1}{p^{e_{1}}}>0$ and $\mathbb{F}_{p^{2}} \subseteq K=K(x, y) v$, from Lemma 5.2 it follows that a root $\vartheta^{\prime}$ of the polynomial $h_{2}$ generates an extension $K\left(x, y, \vartheta^{\prime}\right)$ of $K(x, y)$ contained in the henselization $K(x, y)^{h}$ of $K(x, y)$.

Define $\vartheta=\eta+\vartheta^{\prime} \in \widetilde{K(x, y)}$. By the additivity of the polynomial $Y^{p^{2}}-Y$, the element $\vartheta$ is a root of the polynomial

$$
f:=Y^{p^{2}}-Y-\frac{1}{d^{p}} y-\frac{1}{d^{p^{2}}} y^{p}-\frac{1}{d^{p^{2}}} y^{-n} .
$$

We show that the extension $K(x, y, \vartheta) \mid K(x, y)$ is of degree $p^{2}$. Consider the rational function field $K(y) \mid K$ with the $y$-adic valuation $w$. Then

$$
w \frac{1}{d^{p^{2}}} y^{-n}=p^{e_{1}+2}-n=-1
$$


and $w \frac{1}{d^{p}} y, w \frac{1}{d^{p^{2}}} y^{p}>0$. Hence

$$
w\left(\frac{1}{d^{p}} y+\frac{1}{d^{p^{2}}} y^{p}+\frac{1}{d^{p^{2}}} y^{-n}\right)=-1<0
$$

and consequently $w \vartheta=-\frac{1}{p^{2}}$. Therefore $(w K(y, \vartheta): w K(y)) \geq p^{2}$. On the other hand $[K(y, \vartheta): K(y)] \leq p^{2}$, hence by the fundamental inequality we obtain that $K(y, \vartheta) \mid K(y)$ is of degree $p^{2}$. The element $x$ is transcendental over $K(y)$, thus we have also that

$$
[K(x, y, \vartheta): K(x, y)]=p^{2} .
$$

Since $K(x, y, \eta) \mid K(x, y)$ is linearly disjoint from $K(x, y)^{h} \mid K(x, y)$ and $\vartheta^{\prime} \in K(x, y)^{h}$, from the equality $\vartheta=\eta+\vartheta^{\prime}$ if follows that

$\left[K(x, y)^{h}(\vartheta): K(x, y)^{h}\right]=\left[K(x, y)^{h}(\eta): K(x, y)^{h}\right]=p<p^{2}=[K(x, y, \vartheta): K(x, y)]$.

Therefore $K(x, y, \vartheta) \mid K(x, y)$ is not linearly disjoint from $K(x, y)^{h} \mid K(x, y)$.

On the other hand, the polynomial $f$ can be derived by a deformation of a purely inseparable polynomial inducing an immediate extension which does not lie in the completion of $K(x, y)$ in the following way. Define

$$
\xi:=d^{1-p^{-1}} y^{p^{-2}}-y^{p^{-1}}-y^{-n p^{-2}} .
$$

The value group of $K(x, y)$ is $p$-divisible and the residue field of $K(x, y)$ is perfect, thus $K(x, y, \xi) \mid K(x, y)$ is an immediate purely inseparable extension of degree $p^{2}$. Since dist $\left(y^{1 / p}, K(x, y)\right) \leq 0^{-}$, we have that $v\left(y^{1 / p}-K(x, y)\right)<0$. The values $v d^{1-p^{-1}} y^{p^{-2}}$ and $v y^{-n p^{-2}}$ are positive, therefore also $v(\xi-K(x, y))<0$. It follows that

$$
\operatorname{dist}(\xi, K(x, y)) \leq 0^{-}
$$

and consequently $\xi$ does not lie in the completion of $K(x, y)$. Note that

$$
v \xi=v y^{1 / p}=-\frac{1}{p^{e_{1}+1}}
$$

and thus

$$
\left(p^{2}-1\right) v d+v \xi=p^{2}-1-\frac{1}{p^{e_{1}+1}}>0 \geq p^{2} \operatorname{dist}(\xi, K(x, y)) .
$$

Therefore, from Lemma 5.4 it follows that every root of the polynomial

$$
Y^{p^{2}}-Y-\left(\frac{\xi}{d}\right)^{p^{2}}=Y^{p^{2}}-Y-\frac{1}{d^{p}} y-\frac{1}{d^{p^{2}}} y^{p}-\frac{1}{d^{p^{2}}} y^{-n}=f
$$

is in relation $\sim_{K(x, y)}$ with $\frac{\xi}{d}$. Thus $\vartheta \sim_{K(x, y)} \frac{\xi}{d}$.

Hence by the deformation of the purely inseparable polynomial $Y^{p^{2}}-\xi^{p^{2}}$ we obtain the $p$-polynomial $f=Y^{p^{2}}-Y-\left(\frac{\xi}{d}\right)^{p^{2}}$ generating the extension $K(x, y, \vartheta) \mid K(x, y)$ which is not linearly disjoint from the henselization of $K(x, y)$. The reason why this assertion holds for the extension $K(x, y, \vartheta) \mid K(x, y)$ induced by the polynomial $f$ is that although only the element $\frac{1}{d^{2}} y^{p}$ matters for the approximation of $\xi$ and consequently of $\vartheta$, the elements $\frac{1}{d^{p^{2}}} y^{-n}$ and $\frac{1}{d^{p}} y$ matter for the degrees of the extensions considered in our example. 
As we have seen, the deformation of a polynomial inducing an immediate purely inseparable extension of prime degree not contained in the completion of the field leads to an Artin-Schreier defect extension, because of the relation between the generators of the purely inseparable and the Artin-Schreier extensions. From that relation, by Theorem 2.14, it follows in particular that the Artin-Schreier extension is disjoint from the henselization. Example 5.5 shows that for extensions of higher degrees this implication does not hold. Note that the above example also shows that we cannot generalize Theorem 2.14 replacing the condition " $a$ lies in $K^{h}$ " by " $K(a) \mid K$ is not linearly disjoint from $K^{h} \mid K$ ". Indeed, as we have shown, $\vartheta \sim_{K(x, y)} \frac{\xi}{d}$ and $K(x, y, \vartheta) \mid K(x, y)$ is not linearly disjoint from $K(x, y)^{h} \mid K(x, y)$. On the other hand, the element $\frac{\xi}{d}$ is purely inseparable over $K(x, y)$. It follows that $K\left(x, y, \frac{\xi}{d}\right) \mid K(x, y)$ is linearly disjoint from $K(x, y)^{h} \mid K(x, y)$.

\section{References}

[CP] Cutkosky, D. - Piltant, O.: Ramifcation of valuations, Adv. Math. 183 (2004), 1-79

[E] Endler, O.: Valuation theory, Springer, Berlin (1972)

[Ka] Kaplansky, I. : Maximal fields with valuations I, Duke Math. Journ. 9 (1942), 303-321

[Kn-Ku1] Knaf, H. - Kuhlmann, F.-V. : Abhyankar places admit local uniformization in any characteristic, Ann. Scient. Ec. Norm. Sup. 38 (2005), $833-846$

[Kn-Ku2] Knaf, H. - Kuhlmann, F.-V.: Every place admits local uniformization in a finite extension of the function field, Adv. Math., 221, (2009), 428-453

[Ku1] Kuhlmann, F.-V.: Value groups, residue fields and bad places of rational function fields, Trans. Amer. Math. Soc. 356 (2004), 4559-4600

[Ku2] Kuhlmann, F.-V.: Additive Polynomials and Their Role in the Model Theory of Valued Fields, Proceedings of the Workshop and Conference on Logic, Algebra, and Arithmetic, held October 18-22, 2003. Lecture Notes in Logic 26 (2006), 160-203

[Ku3] Kuhlmann, F.-V.: A classification of Artin-Schreier defect extensions and a characterization of defectless fields, Illinois J. Math. 54 (2010), 397-448

[Ku4] Kuhlmann, F.-V.: Approximation of elements in henselizations, Manuscripta Math. 136 (2011), 461-474

[Ku5] Kuhlmann, F.-V.: The algebra and model theory of tame valued fields, submitted

[Ku6] Kuhlmann, F.-V.: Valuation Theory, book in preparation. Preliminary versions of several chapters are available on the web site: http://math.usask.ca/ fvk/Fvkbook.htm.

[Ku-P] Kuhlmann, F.-V. - Piltant, O.: Higher ramification groups for ArtinSchreier defect extensions, in preparation

[Ku-V] Kuhlmann, F.-V. - Vlahu I.: The relative approximation degree in valued function fields, submitted

[L] Lang, S.: Algebra, Addison-Wesley, New York (1965)

[R] Ribenboim, P.: Théorie des valuations, Les Presses de l'Université de Montréal, Montréal, 1st ed. (1964), 2nd ed. (1968)

[T] Temkin, M.: Inseparable local uniformization, preprint, arXiv:[0804.1554] 
[W] Warner, S.: Topological fields, Mathematics studies 157, North Holland, Amsterdam (1989)

[ZS] Zariski, O. - Samuel, P.: Commutative Algebra, Vol. II, New YorkHeidelberg-Berlin (1960)

University of Silesia, Institute of Mathematics,

Bankowa 14, 40-007 Katowice, Poland

e-mail: ablaszczok@math.us.edu.pl 